\title{
Dynamic dual-isotope molecular imaging elucidates principles for optimizing intrathecal drug delivery
}

\author{
Daniel A. Wolf, Jacob Y. Hesterman, ${ }^{2}$ Jenna M. Sullivan, ${ }^{2}$ Kelly D. Orcutt, ${ }^{2}$ Matthew D. Silva, ${ }^{2}$ \\ Merryl Lobo, ${ }^{2}$ Tyler Wellman, ${ }^{2}$ Jack Hoppin, ${ }^{2}$ and Ajay Verma ${ }^{1}$ \\ ${ }^{1}$ Experimental Medicine, Biogen Inc., Cambridge, Massachusetts, USA. inviCRO LLC, Boston, Massachusetts, USA.
}

\begin{abstract}
The intrathecal (IT) dosing route offers a seemingly obvious solution for delivering drugs directly to the central nervous system. However, gaps in understanding drug molecule behavior within the anatomically and kinetically unique environment of the mammalian IT space have impeded the establishment of pharmacokinetic principles for optimizing regional drug exposure along the neuraxis. Here, we have utilized high-resolution single-photon emission tomography with X-ray computed tomography to study the behavior of multiple molecular imaging tracers following an IT bolus injection, with supporting histology, autoradiography, block-face tomography, and MRI. Using simultaneous dual-isotope imaging, we demonstrate that the regional CNS tissue exposure of molecules with varying chemical properties is affected by IT space anatomy, cerebrospinal fluid (CSF) dynamics, CSF clearance routes, and the location and volume of the injected bolus. These imaging approaches can be used across species to optimize the safety and efficacy of IT drug therapy for neurological disorders.
\end{abstract}

Conflict of interest: D.A. Wolf and A. Verma are employees and shareholders of Biogen Inc. J. Hoppin is a cofounder and managing partner of inviCRO LLC as well as an equity shareholder. J.Y. Hesterman, J.M. Sullivan, K.D. Orcutt, M.D. Silva, M. Lobo, and T. Wellman are employees of inviCRO LLC.

Submitted: October 29, 2015 Accepted: January 27, 2016 Published: February 25, 2016

Reference information: JCI Insight. 2016;1(2):e85311. doi:10.1172/jci.insight.85311.

\section{Introduction}

The development of therapeutics for CNS disorders has been impeded by the selective permeability of the blood-brain barrier (BBB) that isolates the interstitial fluid (ISF) space surrounding CNS cells from the plasma fluid space (1). The ISF is in communication with the cerebrospinal fluid (CSF) via perivascular (Virchow-Robin) routes that connect the CNS parenchyma with the intrathecal (IT) space (also known as subarachnoid or leptomeningeal space) (2-4), making drug delivery into the IT compartment an attractive means for bypassing the BBB. However, use of the lumbar IT dosing route for treating pain and spasticity is known to have high interpatient variability (5-7), and attempts to use this route to deliver therapeutics to more rostral parts of the neuraxis are challenged by significant caudal-to-rostral drug concentration gradients $(8-10)$.

When molecules are introduced into the IT space, their neuraxial exposure may be influenced by their chemical properties, mode of delivery, movement within the CSF, clearance from the CSF into plasma, exchange from the CSF to the ISF, and interaction with CSF proteins and tissue elements (11). Recent biological insights elucidating some of these processes may help establish new principles governing IT drug disposition. For example, CSF convective phenomena resulting from CSF turnover, heartbeat, respiration, and body movement may influence neuraxial spread of IT-dosed drugs (12-15). The glymphatic system of directionally oriented water flux along perivascular spaces may help drive the movement of drug molecules from CSF into ISF $(3,16-18)$. Clearance from CSF to plasma via arachnoid granulations (19-21), as well as via nasal, nerve, or meningeal lymphatics (22-29), may also markedly affect CNS exposure of IT-administered drugs. However, the relatively inaccessible nature of the IT space and the qualitative and technically variable nature of most experimental approaches used to study it have limited our understanding of the relative impact of these mechanisms on specific drug molecules. Molecular positron emission tomography and single-photon emission computed tomography (SPECT) imaging offer the type of sensitivity required for such assessments (11). Here, we have applied multimodal and high-resolution SPECT imaging in concert with histology, autoradiography, MRI, and block-face tomography to elucidate in vivo the combined impact of anatomical, biological, chemical, and pharmacological factors in determining the neuraxial exposure of drug surrogate molecules following an IT lumbar bolus in the rat. 


\section{Results}

Anatomy of the rat subarachnoid space. Both the total volume of CSF within the IT space and regional anatomical expansions of CSF volume (recesses and cisterns) may influence the concentration and distribution of lumbar IT-injected drugs along the neuraxis $(25,26,30,31)$. Live imaging with high-resolution T2-weighted 7T MRI was used to estimate the total CSF volume of age-matched female adult Sprague-Dawley littermates (mean $=562 \mu 1, \mathrm{SD}= \pm 40 \mu 1, n=6$ ). Whole-body MRI allowed us to assess the morphology of the IT space, including identification of anatomical pockets of CSF within both spinal and cranial recesses and cisterns (ref. 32 and Figure 1, A and B).

The distribution of classical pigmented probes in the CSF space following lumbar IT injection was assessed by block-face tomography and digital image reconstruction to create 3D distribution maps (33). Following injection of India ink or Evans blue dye (EBD), serial $25-\mu \mathrm{m}$ whole-body sections were cut, and the tissue block was photographed after each section. Digital Z-stacking of serial images produced a 3D model of the data that allowed viewing from any orientation. IT distribution of colloidal India ink (Figure 1C) followed the general heterogeneous pattern seen for CSF by MRI, with some regional differences attributable to India ink's association with meninges and large vessels (34), as shown by 3D image reconstructions (Figure 1, C-E, and Supplemental Video 1; supplemental material available online with this article; doi:10.1172/jci.insight.85311DS1). Block-face tomography was also used to follow the IT distribution of EBD, a small molecule (MW $961 \mathrm{Da}$ ), which binds tightly to albumin and can move from the CSF into the ISF of CNS tissue (35). Sagittal whole-body images taken 2.5 hours after lumbar IT administration of EBD revealed a caudal-to-rostral gradient in the subarachnoid space along the spinal cord (Figure $1 \mathrm{~F}$ ). Viewing the digital data in axial planes (Figure $1 \mathrm{G}$ and Supplemental Video 2) allowed detection of the dye in the subarachnoid space along the spinal cord (Figure 1G) and also highlighted the caudal-to-rostral gradient of entry into the ISF of sacral, lumbar, thoracic, and cervical spinal cord. These qualitative observations of high concentrations of pigmented molecules demonstrate the complex behaviors of molecules following their introduction into the IT space.

Quantitative drug distribution. Quantitative assessment of drug distribution in the IT space was assessed by high-resolution in vivo imaging using preclinical single-photon emission tomography with X-ray computed tomography (SPECT-CT) with molecular sensitivity in the range of clinically used IT drugs (pM to $\mu \mathrm{M})$. Radiolabeled albumin (MW $66 \mathrm{kDa}$ ) was used as a probe, as it is the predominant protein component in CSF (130-350 mg/1 in humans; ref. 36) and displays low tissue-binding affinity and low cellular entry. SPECT-CT imaging of a 50- $\mu 1$ lumbar IT bolus injection of ${ }^{123}$ I-labeled human serum albumin ( $\left.{ }^{123} \mathrm{I}-\mathrm{HSA}\right)$ at 6 hours after injection resulted in IT retention of nearly all signal (Figure 2A). We observed a severalfold variation in concentration of ${ }^{123} \mathrm{I}-\mathrm{HSA}$ along the neuraxis (Figure 2, A and B), with high exposure at lumbosacral nerve roots and other CSF recesses along the thoracic and cervical cord and within the cranium. Sagittal head and neck images at 2 hours after IT injection confirmed preferential intracranial flow of ${ }^{123}$ I-HSA from the cisterna magna along the ventral brain, through the pituitary recess, and toward the olfactory cisterns simultaneously with flow into both the lateral and medial aspects of the cerebellar cisterns (ref. 16 and Figure 2C). Delayed spread was visualized dorsally within the interhemispheric fissure, with pooling along the dorsal portions of the olfactory cistern (Figure 2C). Upon reaching the olfactory cistern, ${ }^{123}$ I-HSA displayed rapid clearance across the cribriform plate into the nasal turbinates and appeared in the deep cervical nodes by 6 hours after injection (Figure 2D). The small-molecule radiopharmaceutical ${ }^{111}$ In-diethylenetriamine-pentacetic acid ( ${ }^{111} \mathrm{In}-\mathrm{DTPA}$, MW $\left.545 \mathrm{Da}\right)$, which is used clinically for evaluating CSF leaks, showed a similar intracranial distribution pattern to that of ${ }^{123}$ I-HSA at 2 hours following a lumbar bolus injection (30 $\mu$ followed by a $40-\mu 1$ saline flush) (Figure 2E). Ex vivo autoradiography of whole-head sections at this time point confirmed heterogeneous signal movement into brain parenchyma, with the highest amounts associated with pericisternal tissue, as well as movement into olfactory bulbs and nasal turbinates (Figure 2F). Collectively, these data allowed construction of a 3D model of the major rat cranial subarachnoid CSF routes for IT spread of large and small molecules (Figure 2G).

To study differential kinetics of molecules in the IT space, multiple clinically available SPECT radiopharmaceutical probes with different chemical properties were selected as drug surrogates. Distinct radioisotopes were used to tag these molecules, either covalently $\left({ }^{123} \mathrm{I},{ }^{125} \mathrm{I}\right)$ or by radiometal chelation $\left({ }^{99 \mathrm{~m}} \mathrm{Tc}\right.$, ${ }^{111} \mathrm{In}$ ), and at least $95 \%$ radiolabel incorporation was confirmed for all labeling reactions prior to administration. To elucidate the biodistribution of dissociated (free) isotope from the tagged molecules, the pharmacokinetic behavior of $\mathrm{Na}^{123} \mathrm{I},{ }^{111} \mathrm{InCl}_{3}$, or pertechnetate $\left({ }^{99} \mathrm{TcO}_{4}\right)$ alone was determined over 15 minutes 
A

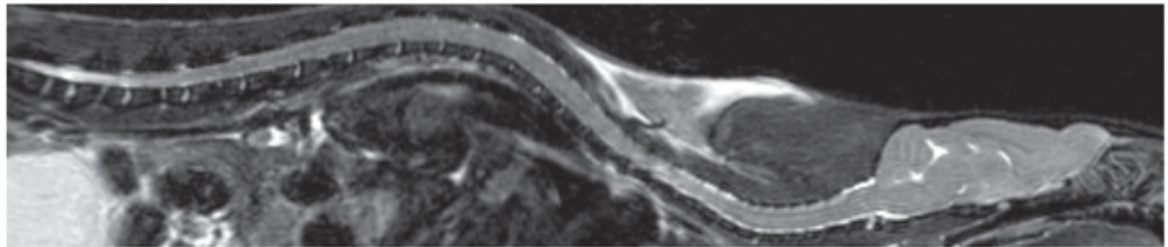

B

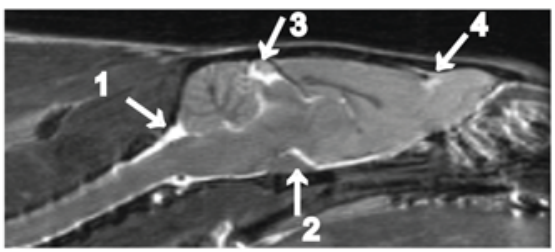

C

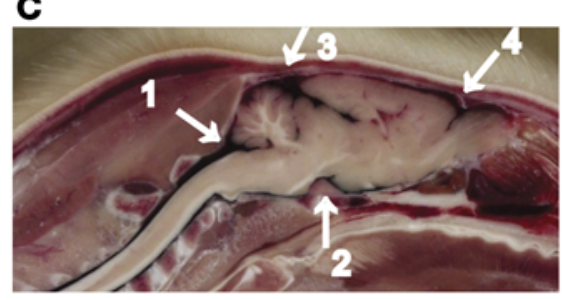

D 1.0

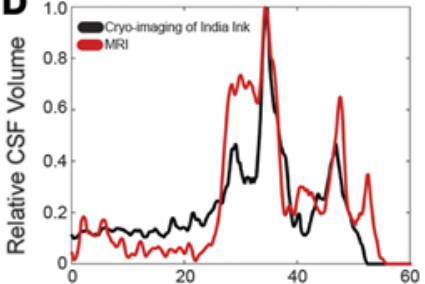

Cervical Spinal Column $\rightarrow$ Front of Cranium $(\mathrm{mm})$

E

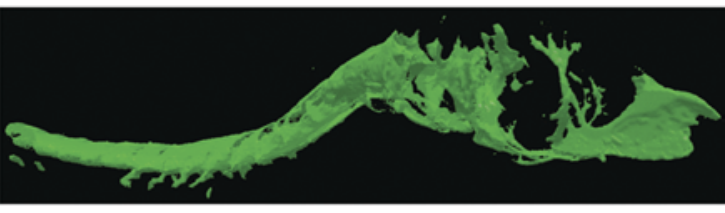

$\mathbf{F}$

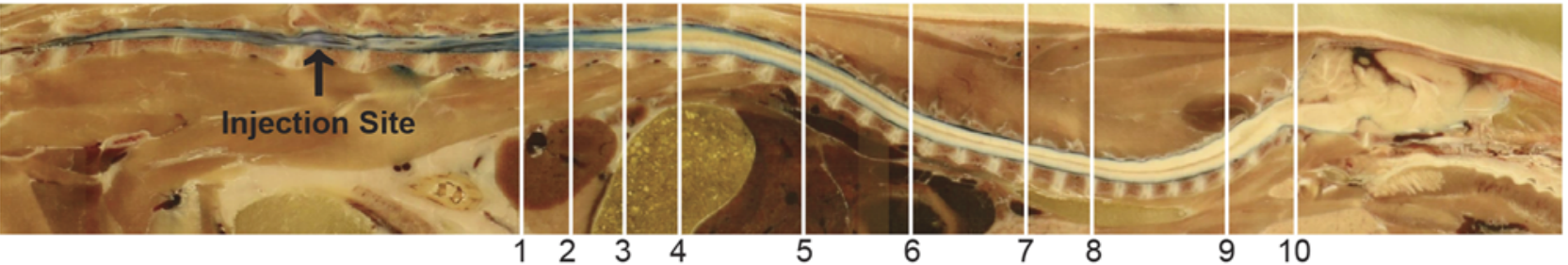

G
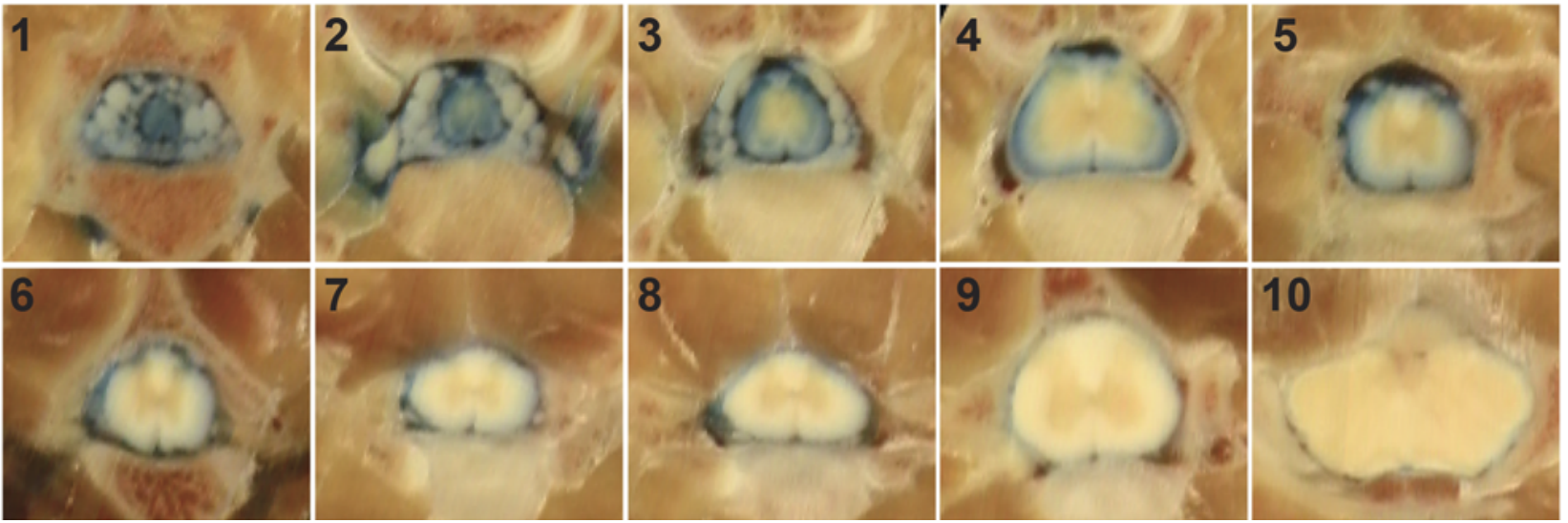

Figure 1. Imaging the intrathecal space by MRI reveals anatomy that closely mirrors that which can be identified using classical dye-imaging techniques. (A) Sagittal high-resolution whole-body T2-weighted MRI image highlighting the intrathecal space along the rostrocaudal neuraxis of the rat (representative image from cohort of $n=6$ ). (B) Focused MRI image of the head, outlining the cranial subarachnoid space in high detail, including major cerebrospinal fluid-containing (CSF-containing) cisterns and recesses (1, cisterna magna; 2, pituitary recess; 3, supracerebellar cistern; 4, olfactory cistern). (C) Sagittal cryosectioned head slice of a rat following lumbar puncture infusion of India ink ( $n=1$ experiment performed), emphasizing congruent intrathecal anatomy to that of CSF imaged by magnetic resonance (see Supplemental Video 1 for 3D reconstruction of serial sections). (D) Comparison of CSF volume measurement calculated from a 3D reconstruction of serial cryosections of a rat following lumbar intrathecal injection of India ink $(n=1)$ and by whole-body T2-weighted MRI $(n=1)$. (E) 3D rendering of the India ink, highlighting the intrathecal space within the cervical spinal column and cranium. Note the pockets of CSF branching out along cervical nerve roots, running along the ventral aspects of the brain and along lateral aspects of the supracerebellar and olfactory cisterns as well as the middle cerebral artery. (F) Sagittal whole-body cryosection of a rat following lumbar intrathecal injection of Evans blue dye (representative image from $n=2$ experiments performed). (C) Cross-sectional views of the Evans blue dye-filled intrathecal space at different levels of the spinal column, as rendered from a 3D reconstruction made from serial sagittal cryosections, as in E (see Supplemental Video 2 for fly-through view of the 3D reconstruction). Note the rostral to caudal gradient of ink within the intrathecal space as well as exchange into the spinal cord interstitial fluid. Original magnification, $\times 1$ (A and F); ×1.5 (B, C, and E); and x4 (G). 
A
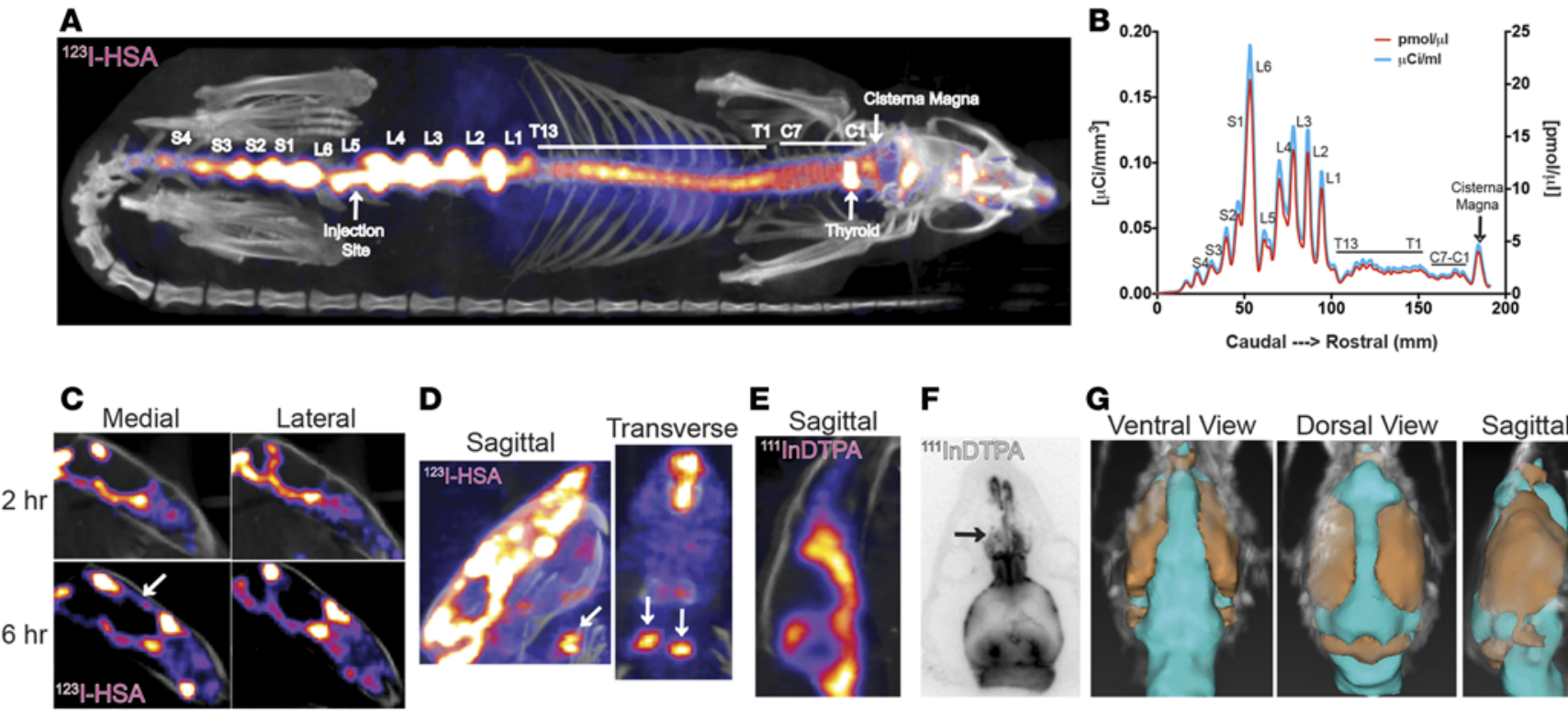

D
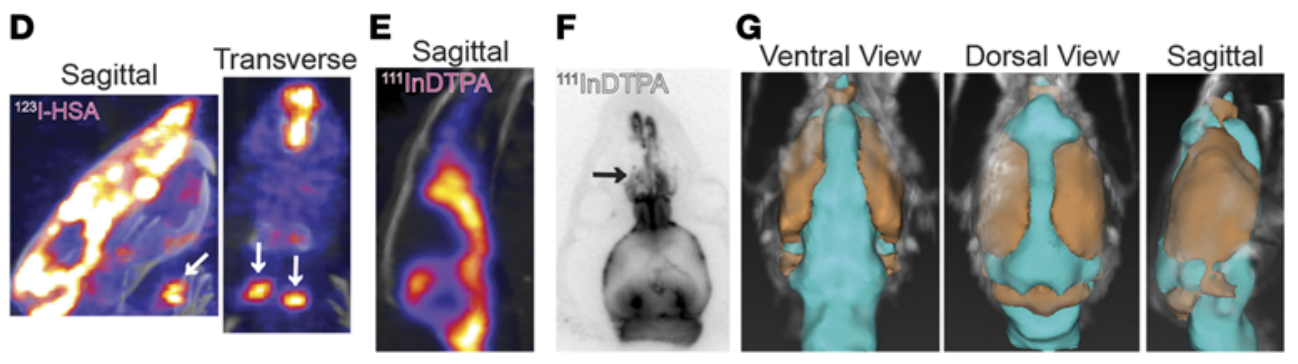

Figure 2. Noninvasive single-photon emission tomography with X-ray computed tomography imaging can be used to quantitatively track the biodistribution of molecules within the intrathecal space with high resolution. (A) High-resolution single-photon emission tomography with X-ray computed tomography (SPECT-CT) imaging of a rat (representative from a cohort of $n=5$ ) 6 hours after lumbar injection of $50 \mu$ of ${ }^{123}$-labeled human serum albumin (123I-HSA), showing radiotracer accumulation within cerebrospinal fluid-containing (CSF-containing) lacunes in the intrathecal space along the neuraxis, most notably adjacent to the lumbar nerve roots. (B) Quantification of radiotracer concentration along the neuraxis, within the spinal intrathecal space region of interest (ROI) from the animal shown in A. Peaks and troughs correspond to CSF pockets along anatomical nerve routes between vertebrae (anatomical locations labeled along the plots). (C) High-resolution SPECT-CT sagittal head images of ${ }^{123}$ I-HSA following a 50- $\mu$ l lumbar intrathecal injection of the radiotracer (representative images from a cohort of $n=5$ ) revealed faster kinetics of solute transport through the CSF along the ventral rather than the dorsal surface of the brain. 2 hours after injection, the tracer had flowed rostrally along the ventral surface of the brain, through the pituitary recess and toward the olfactory cisterns as well as laterally along and into the supracerebellar cistern. Later, at 6 hours after injection, the tracer appeared along the medial interhemispheric fissure of the dorsal surface of the cerebral cortex (arrow). (D) 6 hours after injection of a 50- $\mu$ l bolus of ${ }^{123}$-HSA, tracer accumulation was observed across the cribriform plate in the nasal lymphatics and had accumulated within cervical lymph nodes (arrows). (E) Intrathecal injection of the small-molecule ${ }^{111}$ In-diethylenetriamine-pentacetic acid (111In-DTPA) (representative image from a cohort of $n=5$ ) resulted in a similar pattern of distribution in the cranial intrathecal space 2 hours after injection. (F) Autoradiogram of a slice through the transverse plane of the head of a rat 2 hours after lumbar infusion of ${ }^{111}$ In-DTPA ( $n=1$ experiment performed) depicts outflow of the imaging agent from the CSF into the nasal lymphatics (arrow). (G) A 3D model of the main routes of molecule movement through the cranial CSF constructed from multiple SPECT-CT scans of lumbar intrathecally administered ${ }^{123}$ I-HSA 6 hours after injection. (blue = routes of molecule trafficking, light brown = brain parenchyma). Original magnification, $\times 0.5(\mathbf{C}) ; \times 0.75(\mathbf{D}-\mathbf{F}) ;$ and $\times 1$ (G).

following either i.v. or IT dosing (Supplemental Figure 1, A, C, and E). Each isotope displayed a characteristic i.v. biodistribution pattern, with both ${ }^{123} \mathrm{I}$ and ${ }^{99} \mathrm{mcO}_{4}$ showing uptake by the thyroid gland, gastric epithelium, and nasal epithelium and ${ }^{111} \mathrm{InCl}_{3}$ showing a prominent vascular signal, rapid renal clearance, and bladder accumulation. Following lumbar IT injection, all 3 isotopes displayed rapid clearance from the IT space within the first 15 minutes of injection and subsequently adopted their characteristic i.v. peripheral organ accumulation patterns. When conjugated to other molecules, the signal from these isotopes behaved differently. No leakage to the periphery was seen at either 15 or 60 minutes with IT-injected ${ }^{123}$ I-HSA (Supplemental Figure 1B). A mixed pattern was observed for IT-dosed ${ }^{111}$ In-DTPA, with rapid signal appearance in the kidney and bladder in addition to prolonged retention within the IT space (Supplemental Figure 1D). Surprisingly, the small-molecule radiopharmaceutical ${ }^{99 \mathrm{~m}} \mathrm{Tc}$-dimercaptosuccinic acid ${ }^{99 \mathrm{~m}} \mathrm{Tc}-\mathrm{DMSA}$, MW $492 \mathrm{Da}$ ) showed slower clearance from the IT space as compared to ${ }^{111} \mathrm{In}-\mathrm{DTPA}$, more closely resembling the behavior of ${ }^{123} \mathrm{I}$-HSA (Supplemental Figure 1F).

To empirically characterize the differential IT clearance of these distinct molecules following a lumbar bolus, a dual-isotope rapid live planar scintigraphy technique was used that allowed us to observe two molecules simultaneously following coadministration. IT injection of a mixed solution of two different molecular weight tracers, ${ }^{99} \mathrm{TcO}_{4}\left(\mathrm{MW} 162 \mathrm{Da}\right.$ ) and ${ }^{125} \mathrm{I}$-HSA (MW $66 \mathrm{KDa}$ ), was followed by dynamic imaging of each isotope using distinct radiation energy windows $\left(24.3-29.7 \mathrm{keV}\right.$ for ${ }^{125} \mathrm{I}$ vs. $126.5-154.6 \mathrm{keV}$ for ${ }^{99 \mathrm{~m} T c}$; Supplemental Figure 2A). Both agents spread along the neuraxis rapidly after bolus injection (Figure $3 \mathrm{~A}$ and Supplemental Video 3), with ${ }^{99} \mathrm{TcO}_{4}$ readily clearing from the IT space within the first 10 minutes 
after injection and accumulating in peripheral organs, such as the stomach epithelium, kidneys, and bladder (Figure 3A, middle). Meanwhile, ${ }^{125}$ I-HSA remained contained within the IT space and, following the initial rapid spread from the bolus, continued to move along the neuraxis at a slower average rate, on the order of approximately $1.5 \mathrm{~cm} / \mathrm{h}$ (Figure 3A, top, and Supplemental Video 3). In separate experiments, we did not observe significant binding of ${ }^{99} \mathrm{TcO}_{4}$ to rat or human CSF protein but did observe some binding to HSA. Thus, although ${ }^{99} \mathrm{TcO}_{4}$ and ${ }^{125} \mathrm{I}-\mathrm{HSA}$ clearly displayed differential IT pharmacokinetic and retention profiles (Figure 3, D and E), the coinjection experiments likely underestimate the leakiness of ${ }^{99} \mathrm{TcO}_{4}$ from the IT space.

Dynamic dual-isotope imaging was also used to study the behavior of ${ }^{111} \mathrm{In}$-DTPA and ${ }^{99 \mathrm{~m}}$ Tc-DMSA following IT administration (Figure 3B). Although both are small hydrophilic molecules, ${ }^{99 \mathrm{~m}} \mathrm{Tc}-\mathrm{DMSA}$ exhibits higher plasma protein binding than ${ }^{99 m}$ Tc-DTPA $(37,38)$ and was observed to exhibit greater protein-binding affinity for both rat and human CSF as well as HSA (Figure 3C). Dual-isotope planar SPECT imaging of a mixture of both molecules following IT bolus showed that the higher protein binding ${ }^{99 \mathrm{~m}} \mathrm{Tc}$-DMSA was retained within the IT space to a greater extent than ${ }^{111} \mathrm{In}$-DTPA, with biodistribution kinetics different from ${ }^{99} \mathrm{~m}_{\mathrm{TcO}}$ but similar to that of ${ }^{123} \mathrm{I}$-HSA or ${ }^{125} \mathrm{I}$-HSA (Supplemental Figure 1F; Supplemental Figure 3, B and F; and Supplemental Video 4). These qualitative and quantitative analyses reveal the impact of molecular weight and protein binding on IT drug clearance from the IT space to peripheral tissues.

Effect of IT bolus volume. The effect of bolus injection volume on distribution of molecules following IT administration (11) was empirically investigated using equivalent doses of ${ }^{123} \mathrm{I}$-HSA given in two different bolus volumes $(10 \mu \mathrm{l}$ or $50 \mu \mathrm{l})$. When approximately $700 \mu \mathrm{Ci}$ of ${ }^{123}$ I-HSA was injected as a $10-\mu 1$ bolus (equivalent to $1.8 \% \pm 0.2 \%$ of the total rat CSF volume, as calculated by MRI), the majority of the dose initially filled the IT space within the lumbar and thoracic regions of the spinal column within the first minutes following the injection (Figure 4, A and B). Over time, the ${ }^{123}$ I-HSA slowly moved bidirectionally along the neuraxis, with the leading edge of the radiotracer extended up through the cervical region of the spinal column to the foramen magnum by 2 hours. As shown in Figure 2, A-D, the ${ }^{123}$ I-HSA was widely spread throughout the cranial CSF spaces by 6 hours and had begun to enter the nasal lymphatics. When the same dose was administered as a larger $50-\mu 1$ bolus $(8.9 \% \pm 0.7 \%$ of total CSF volume), the radiotracer initially spread through the entire expanse, from the lumbar to cervical regions, in the first 15 minutes after injection (Figure 4, A and B). After 2 hours, ${ }^{123}$ I-HSA had already filled the basal and olfactory cisterns surrounding the brain and was observed crossing the nasal cribriform plate. Time-activity curves comparing the proportion of radiotracer present within lumbar, thoracic, and cervical cord regions of interest (ROIs) highlight the marked rostral shift in drug distribution with increased bolus volume (Figure 4C). AUC values (see the legend for Figure 4C) determined from these curves also demonstrated a marked rostral shift as well as a reduction in local injection site exposure.

Influence of tissue-binding affinity on distribution of intrathecally administered drugs. Lumbar IT dosing of drugs with strong CNS tissue affinity may lead to high, potentially toxic, local spinal cord exposure, with minimal spread to more rostral brain regions. To study this variable, we utilized ${ }^{99 \mathrm{~m}} \mathrm{Tc}$-sestamibi (MW $777.85 \mathrm{Da})$, a clinical cardiac imaging agent that enters cells and becomes trapped within active mitochondria (12). As expected, i.v. dosing of ${ }^{99 \mathrm{~m}} \mathrm{Tc}$-sestamibi resulted in accumulation within cardiac tissue (Figure $5 \mathrm{~A})$ and displayed expected hepatic clearance to the gastrointestinal tract. Unlike albumin, a 10- $\mu 1$ lumbar IT dose of ${ }^{99} \mathrm{~m}$ Tc-sestamibi was retained within the IT space without significant spread along the neuraxis between 2 and 6 hours after administration (Figure 5B). Local retention by spinal cord tissue was suggested by autoradiograms, which were grainy due to the relatively low energy $(\gamma=140 \mathrm{keV})$ and short half-life (6 hours) of ${ }^{99 \mathrm{~m}} \mathrm{Tc}$ (Figure $5 \mathrm{C}$ ). Spinal cord tissue uptake was more clearly confirmed by IT catheter injection of higher-energy (mean $\beta=0.34 \mathrm{MeV}, \gamma=186 \mathrm{keV}$ [9\%]) and longer half-life (3.7 days) ${ }^{186}$ Re-sestamibi. SPECT-CT imaging for 6 hours showed that the agent did not display significant caudal-to-rostral IT movement over time (Figure 5D), and high-resolution microautoradiography confirmed localization of ${ }^{186} \mathrm{Re}-$ sestamibi to the spinal cord gray matter (Figure 5E).

To directly compare IT drug distribution of ${ }^{111}$ In-DTPA and ${ }^{99 \mathrm{~m}} \mathrm{Tc}$-sestamibi, a mixture of the two agents was coadministered in a $20-\mu 1$ volume $(3.6 \% \pm 0.2 \%$ of total CSF volume) via lumbar IT bolus injection followed by dual-isotope SPECT-CT imaging. ${ }^{111} \mathrm{In}$-DTPA slowly traveled up the neuraxis and filled the cranial subarachnoid space within 6 hours (Figure 5F and Supplemental Video 5). Concurrently, a progressive decline of tracer concentration in caudal lumbar regions was observed over time (Figure 
A
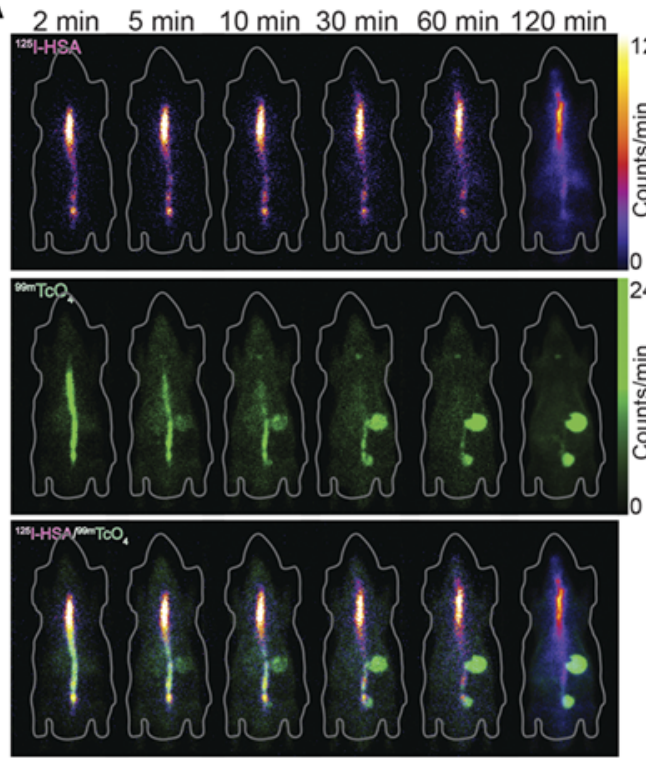

B
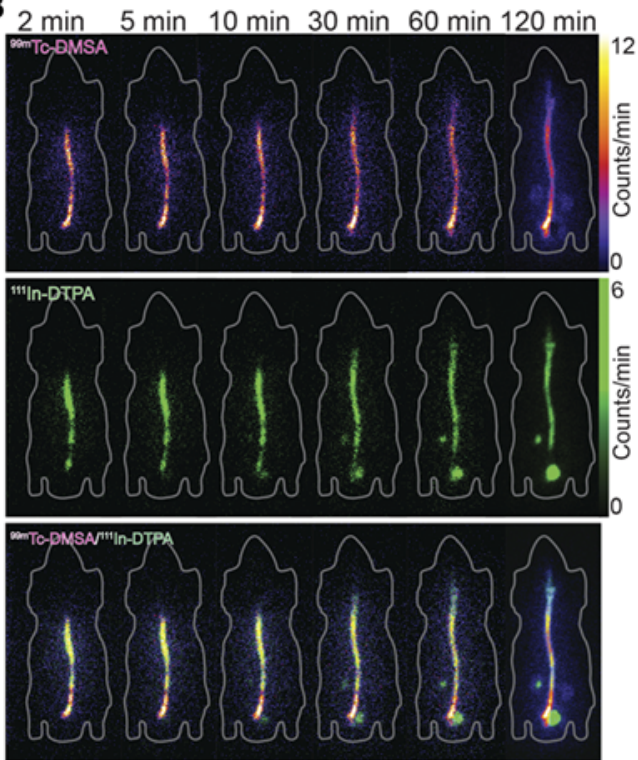

C

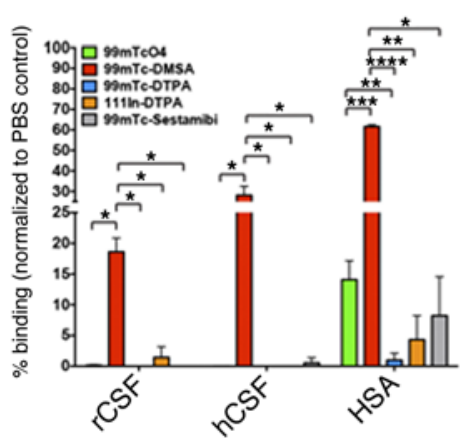

F

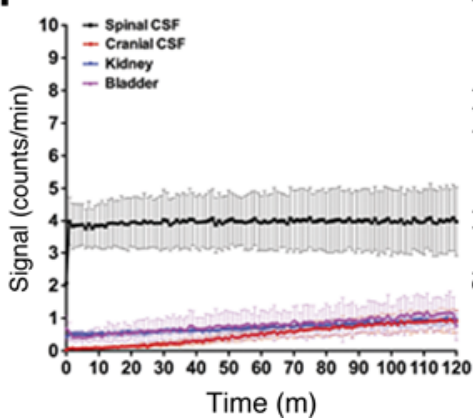

D

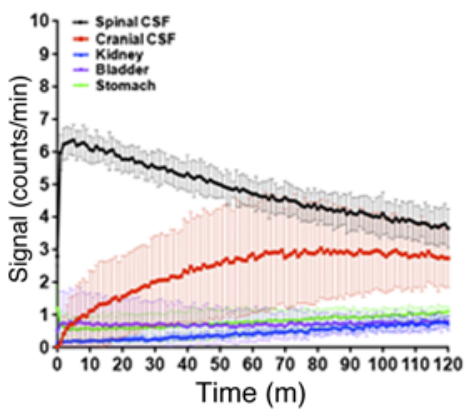

G

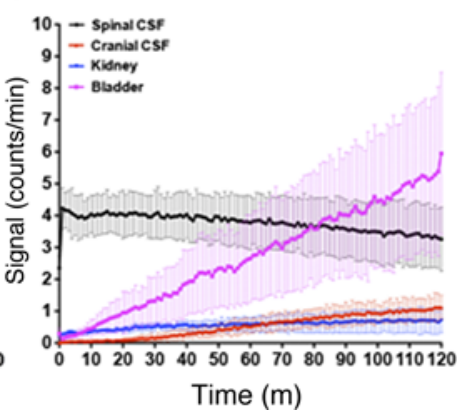

Figure 3. Molecular weight and protein-binding affinity affect both the distribution and retention of molecules within the intrathecal space. (A and B) Simultaneous dynamic dual imaging of (A) ${ }^{123}$ |-labeled human serum albumin $\left({ }^{123}\right.$ I-HSA) and pertechnetate $\left({ }^{99 \mathrm{~m}} \mathrm{TcO}_{4}\right)$ (representative data shown from 1 animal of a cohort of $n=4$ ) and (B) ${ }^{99 m} \mathrm{mc}$-dimercaptosuccinic acid ${ }^{\left({ }^{99 m} T c-D M S A\right.}$ ) and ${ }^{11}$ In-diethylenetriamine-pentacetic acid ${ }^{1{ }^{11}}$ In-DTPA) (representative data shown from 1 animal of a cohort of $n=4$ ) using planar scintigraphy following injection of a mixture of the two molecules. Snapshot images were created from 1-minute-long scanning windows at 2, 5, 10, 30, and 60 minutes and a 10-minute-long scanning window 120 minutes after

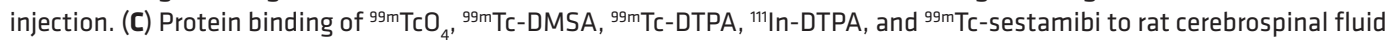
(CSF) ( $r C S F)$, human CSF (hCSF), and HSA. Each experiment was performed in triplicate, and data are depicted as the mean \pm SD. ${ }^{*} P<0.05,{ }^{* *} P<0.01,{ }^{* *} P<0.001,{ }^{* * *} P<0.0001$, 1-way ANOVA followed by Tukey's multiple comparisons test. (DG) Quantitative analysis of the planar scintigraphy data presented in $\mathbf{A}$ and $\mathbf{B}$ represented as time-activity curves, depicting the detectable radioactive counts from (D) ${ }^{125} \mathrm{I}-\mathrm{HSA},(\mathbf{E}){ }^{99 \mathrm{~m}} \mathrm{TcO}_{4},(\mathbf{F}){ }^{99 \mathrm{~m}} \mathrm{Tc}-\mathrm{DMSA}$, and (C) ${ }^{111} \mathrm{In}-\mathrm{DTPA}$ within the chosen regions of interest. Data are presented as the mean \pm SD. 
A
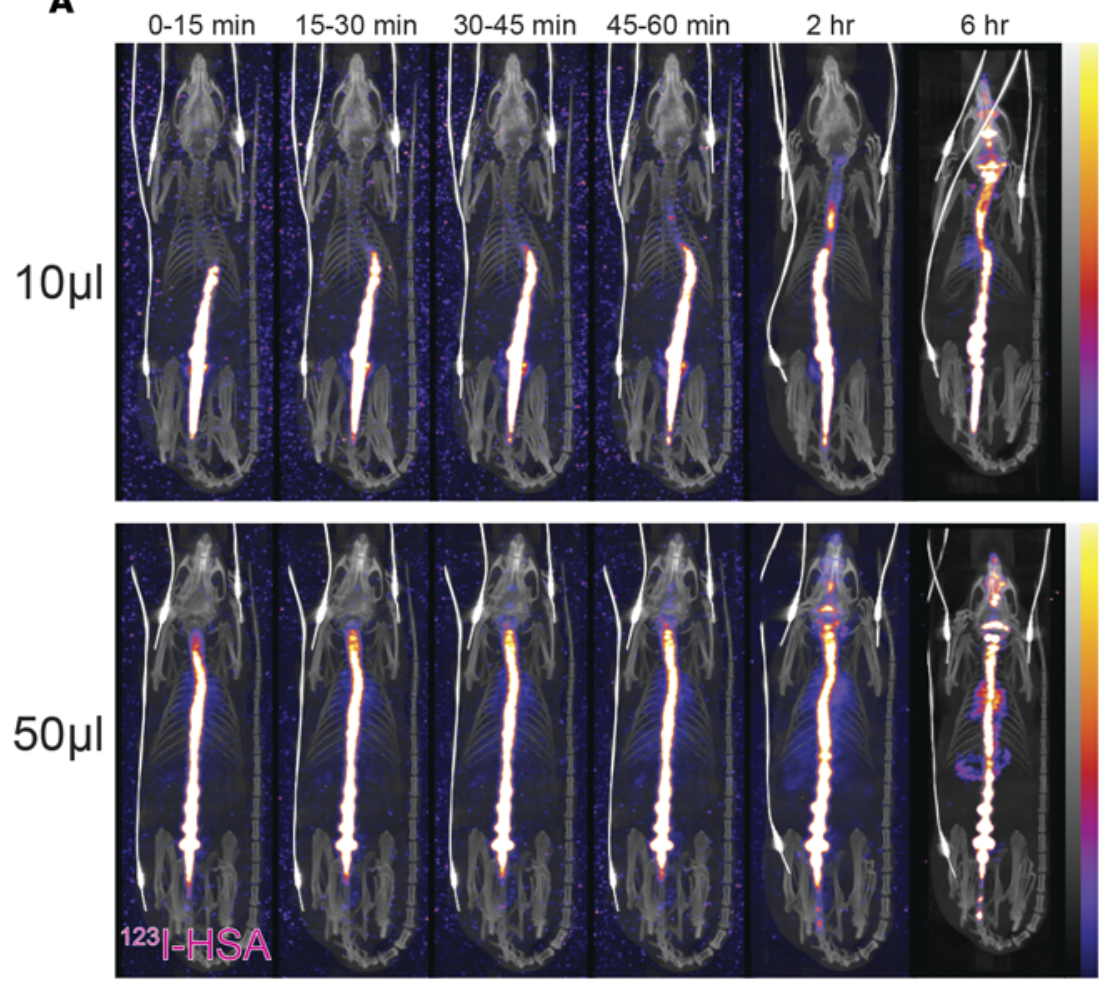

B

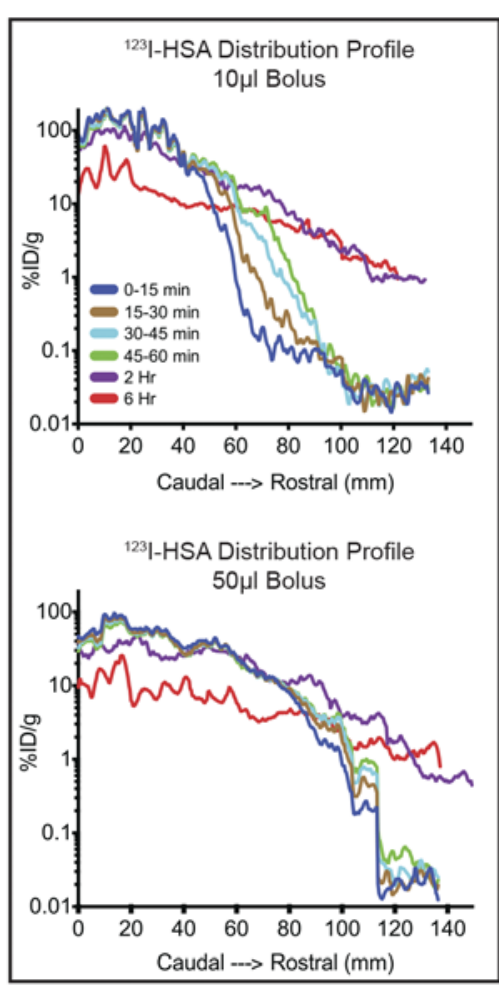

C

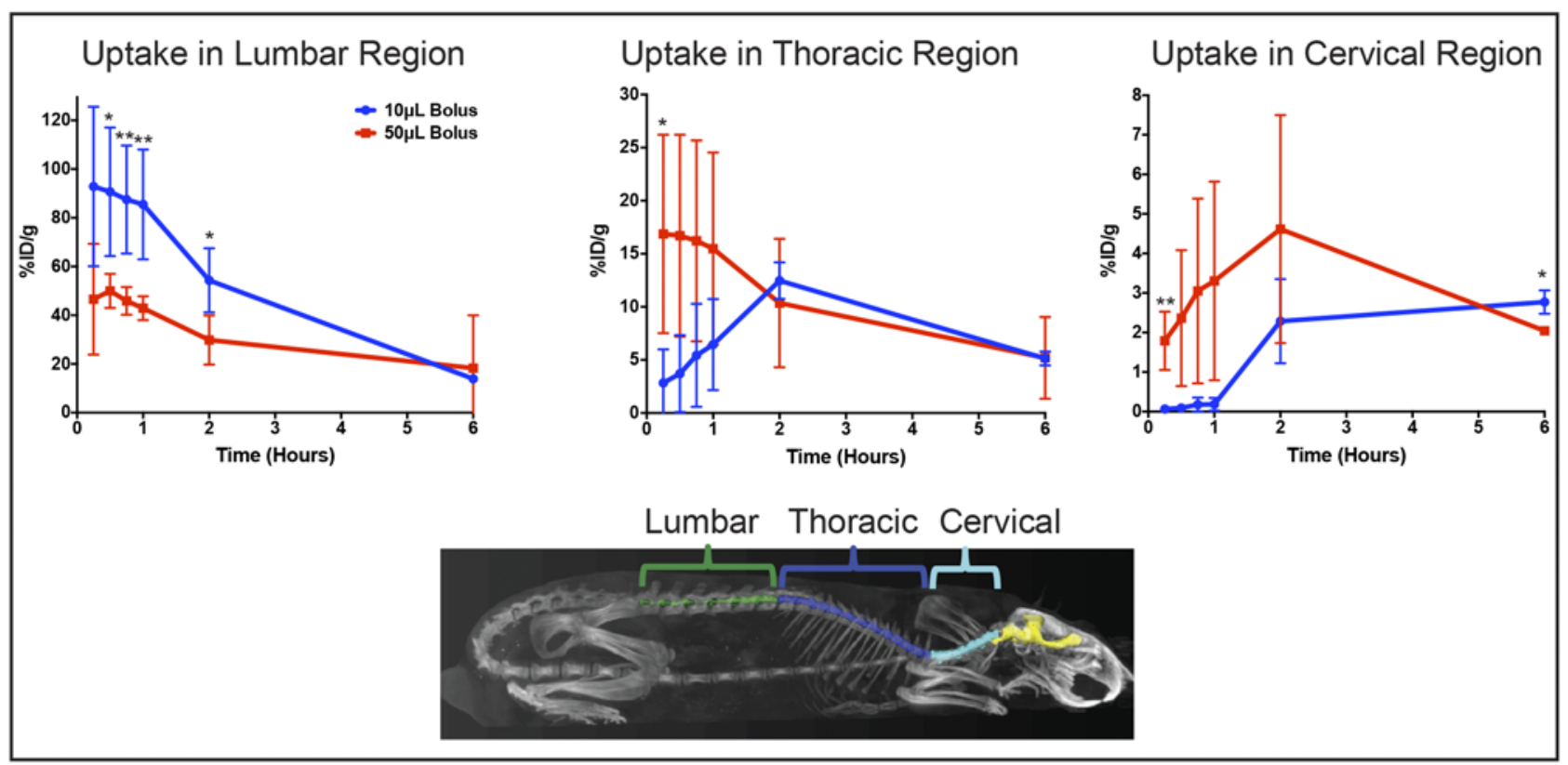

Figure 4. Effect of bolus volume on rostral drug distribution following intrathecal lumbar administration. (A) Single-photon emission tomography with X-ray computed tomography images from representative animals demonstrating ${ }^{123}$ |-labeled human serum albumin (123/-HSA) distribution along the rostrocaudal neuraxis following either a $10-\mu \mathrm{l}$ or $50-\mu \mathrm{l}$ bolus injection of roughly equivalent amounts of tracer. Note that, due to radioactive decay of ${ }^{123} \mathrm{I}$ over time, images are all scaled differently in order to allow for easier visual interpretation. (B) A graphical depiction of the distribution of ${ }^{123}$ I-HSA, within a region of interest (ROI) representative of the intrathecal space, spread along the rostrocaudal neuraxis over time. The graphs represent average values from multiple animals ( $n=4$ for $10 \mu$ infusion, $n=5$ for $50 \mu$ infusion). (C) Time-activity curves demonstrating ${ }^{123}$-HSA uptake in the cervical, thoracic, and lumbar regions of the spinal column over time and a depiction of ROls used for these analyses. Data points represent the mean $\pm \mathrm{SD} .{ }^{*} P<0.05,{ }^{*} P<0.01$, unpaired 2-tailed Student's $t$ test. 2 -way repeated-measures ANOVA analyses revealed significant differences in overall uptake of ${ }^{123}$-HSA into the cervical $(P<0.001)$, thoracic $(P<0.01)$, and lumbar $(P<0.0001)$ regions of interest (ROIs) over the time course of the experiment attributable to the difference in the volume of bolus administered between the two groups. Mean AUC values: (lumbar: $10 \mu l=273$, $50 \mu \mathrm{l}=168$ ), (thoracic: $10 \mu \mathrm{l}=48.1,50 \mu \mathrm{l}=56.3$ ), (cervical: $10 \mu \mathrm{l}=11.5,50 \mu \mathrm{l}=19.3$ ). 
A Intravenous

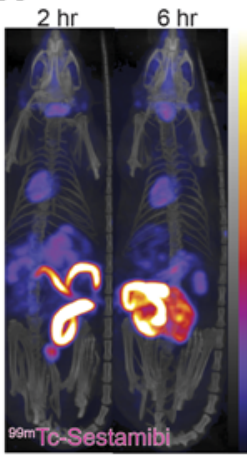

$\mathbf{F}$
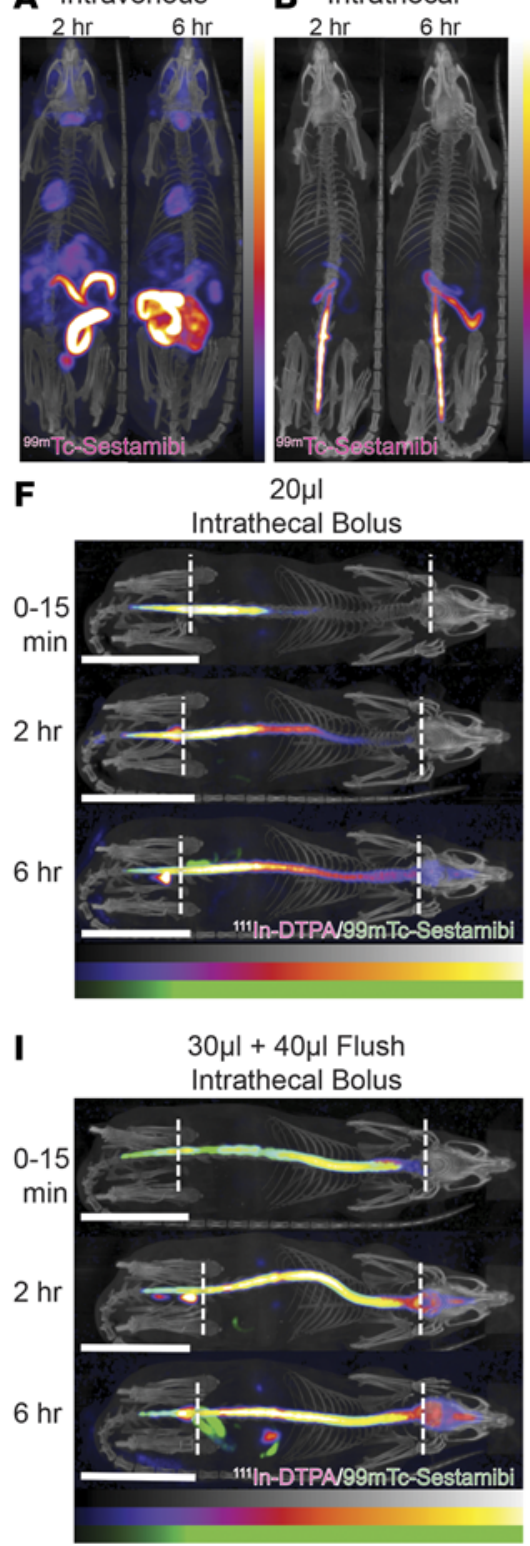

$20 \mu$

ecal Bolus
C

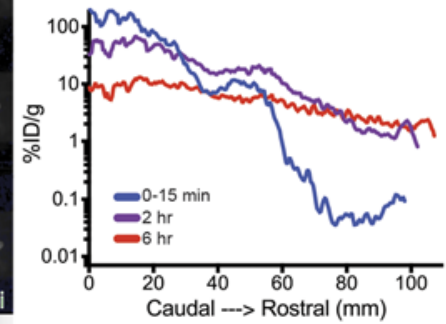

J

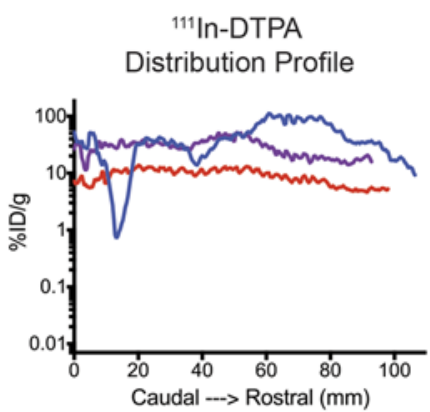

E
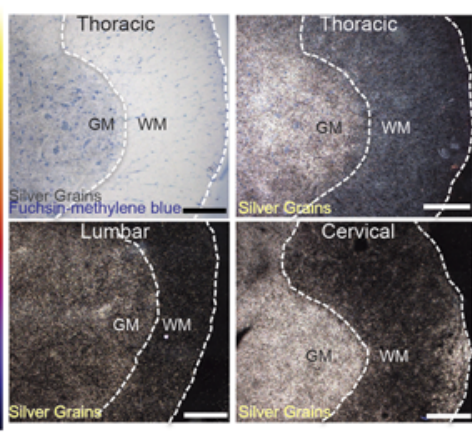

H

99mTc-Sestamibi

Distribution Profile

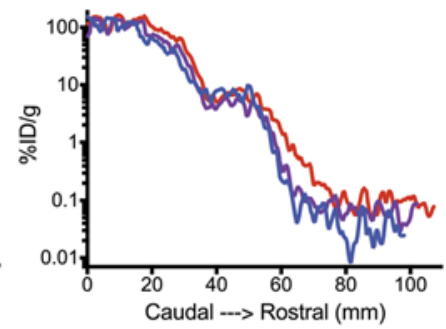

$\mathbf{K}$

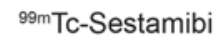

Distribution Profile

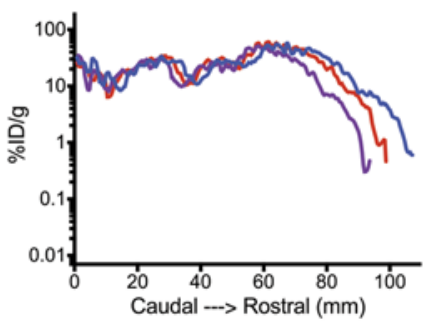

Figure 5. Effect of tissue affinity of a drug on rostral drug distribution following intrathecal lumbar administration. (A) Static single-photon emission tomography with X-ray computed tomography (SPECT-CT) images of ${ }^{99 m}$ Tc-sestamibi 2 hours and 6 hours after intravenous infusion of the tracer into a rat $(n=1$ experiment conducted). (B) Static SPECT-CT images of ${ }^{99 \mathrm{~m} T C} \mathrm{Tc}$-sestamibi 2 hours and 6 hours after $10-\mu$ l lumbar intrathecal bolus injection (representative image from cohort of $n=2$ ). (C) Autoradiogram of a whole-body transverse slice through the lumbar region of the spinal column (black arrow) 2 hours after intrathecal injection of ${ }^{99 m} \mathrm{Tc}$-sestamibi ( $n=1$ experiment conducted). Original magnification, $\times 1$. (D) Static SPECT-CT images of ${ }^{186}$ Re-sestamibi 2 hours and 6 hours after intrathecal bolus infusion of $40 \mu$ of the tracer into an intrathecally catheterized rat (representative image from cohort of $n=2$ ). (E) Microautoradiograms of transverse spinal cord sections from a separate animal that was similarly dosed intrathecally with ${ }^{186} \mathrm{Re}$-sestamibi via a $40-\mu \mathrm{l}$ plus 30- $\mu \mathrm{l}$ saline flush bolus (representative image from cohort of $n=2$ ). Top: Images of a microautoradiogram from a thoracic spinal cord section. Top left: As visualized by bright-field microscopy. Top right: As visualized by darkfield microscopy. Bottom: A section from the lumbar (left) and cervical (right) region of the spinal cord, as visualized by dark-field microscopy. WM, white matter; CM, gray matter. Scale bars: $100 \mu \mathrm{m}$. (F and I) Static SPECT-CT images of ${ }^{11}$ In-diethylenetriamine-pentacetic acid ('11'In-DTPA) (green scale) and ${ }^{99 \mathrm{~m}} \mathrm{Tc}$-sestamibi (fire scale) 0-15 minutes, 2 hours, and 6 hours after lumbar injection of a mixture of the two tracers given as either a (F) $20-\mu \mathrm{I}(n=6)$ or (I) $30-\mu \mathrm{l}$ plus $40-\mu \mathrm{l}$ saline flush bolus $(n=5)$. Images are from 1 representative animal from each group. Scale bars: $50 \mathrm{~mm}$. Vertical dotted lines represent the rostral and caudal boundaries of the intrathecal space region of interest created for quantitative

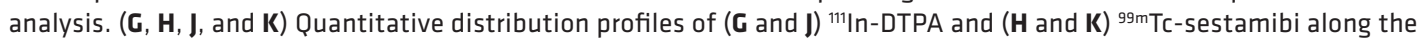
neuraxis from the data from the representative animals presented in $\mathbf{F}$ and $\mathbf{I}$. 
A

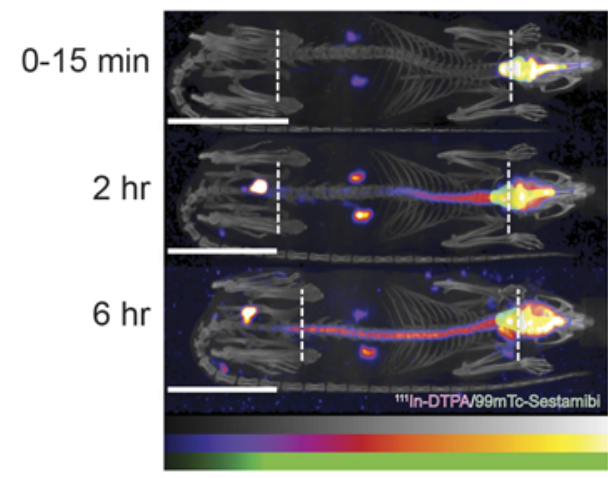

D
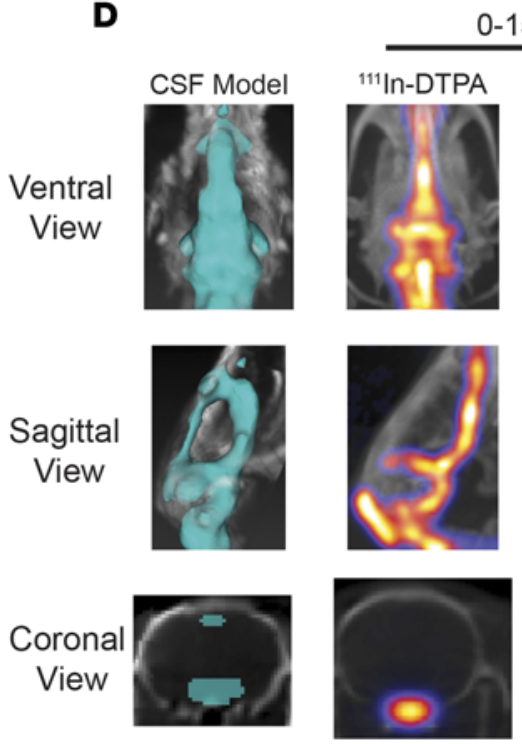

B

\section{${ }^{111}$ In-DTPA Distribution Profile}

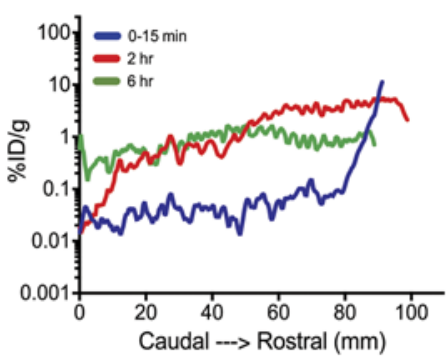

$-15 \min$
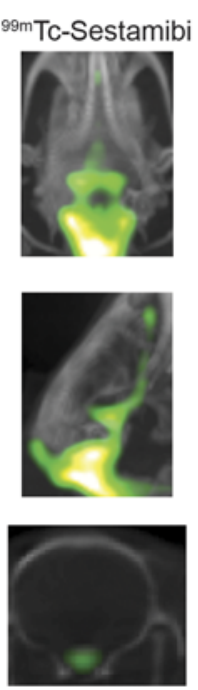

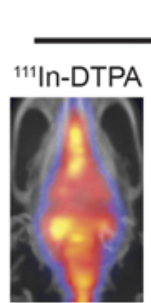

$2 \mathrm{hr}$
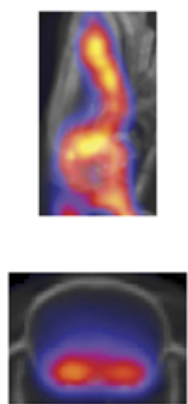

C

\section{${ }^{99 m}$ Tc-Sestamibi Distribution Profile}
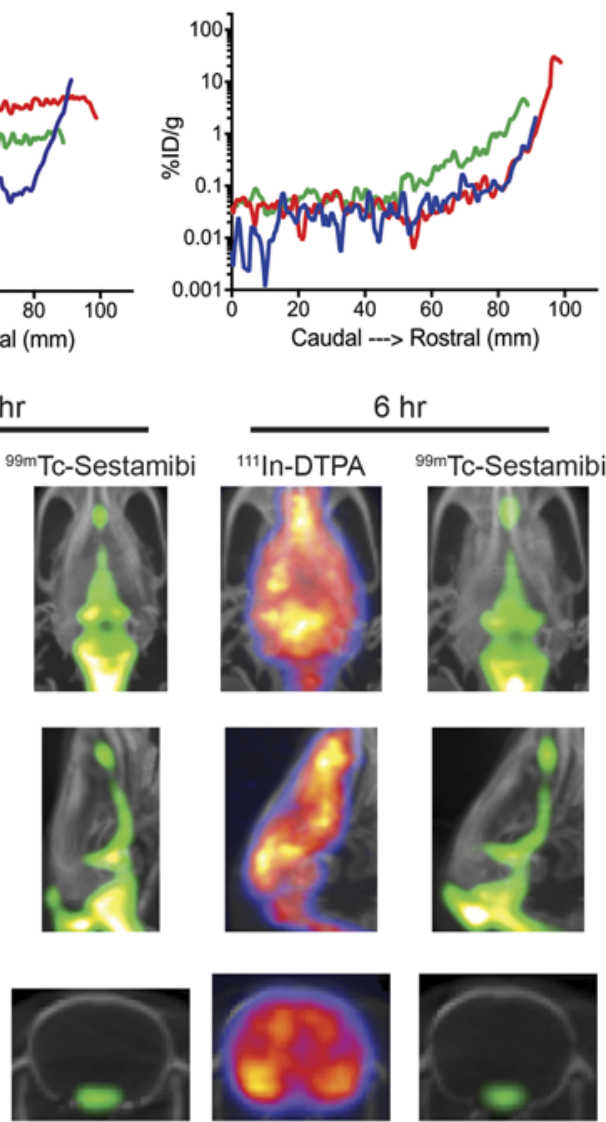

Figure 6. Application of dual single-photon emission tomography with X-ray computed tomography imaging to monitor differential cerebrospinal fluid-interstitial fluid exchange of drug surrogate molecules. (A) Static single-photon emission tomography with X-ray computed tomography (SPECT-CT) images of ${ }^{111}$ In-diethylenetriamine-pentacetic acid ("11'In-DTPA) (green scale) and ${ }^{99 \mathrm{~m} T c-s e s t a m i b i}$ (fire scale) 0-15 minutes, 2 hours, and 6 hours after intracisternal injection of a $10-\mu l$ mixture of the two tracers. Images are from a representative animal from a cohort of $n=4$. (B and $\mathbf{C}$ ) Graphical representations of

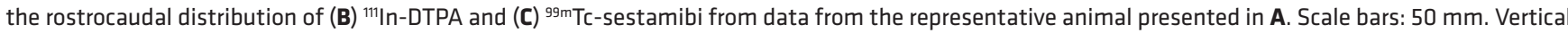
dotted lines represent the rostral and caudal boundaries of the intrathecal space region of interest created for quantitative analysis. (D) Close-up cranial images of data presented in $\mathbf{A}$ in order to highlight the penetration from the cerebrospinal fluid (CSF) into the interstitial fluid of the brain parenchyma. ${ }^{111}$ In-DTPA was observed penetrating into the interstitial fluid of the brain parenchyma over the time course of the experiment. In contrast, ${ }^{99 \mathrm{~m}} \mathrm{Tc}-\mathrm{sestamibi}$ displayed minimal movement from the CSF to the brain parenchyma over a 6-hour time period. Original magnification, $\times 0.75$.

5G and Supplemental Figure 3). Meanwhile, ${ }^{99 \mathrm{~m}} \mathrm{Tc}$-sestamibi remained contained within the lumbar and thoracic cord throughout the duration of the study and displayed little movement up the spinal canal over time (Figure 5, F and H, and Supplemental Figure 3). To test whether increasing the bolus volume would reduce the local sequestration of high-tissue-affinity molecules and improve their distribution to rostral structures, $30 \mu 1$ of a mixture of ${ }^{111} \mathrm{In}$-DTPA and ${ }^{99 \mathrm{~m}} \mathrm{Tc}$-sestamibi was delivered followed immediately by a $40-\mu 1$ saline flush, separated by an air bubble $(70-\mu 1$ total or $12.5 \% \pm 0.9 \%$ of total CSF volume). ${ }^{111}$ In-DTPA immediately filled the subarachnoid space of the entire spinal canal up to the level of the foramen magnum and flowed into the cranial CSF spaces within 2 hours (Figure 5I and Supplemental Video 5). ${ }^{99 \mathrm{~m}} \mathrm{Tc}$-sestamibi also immediately filled the subarachnoid space of the entire spinal column but did not travel further rostrally over time. Quantitative distribution profiles of both ${ }^{111}$ In-DTPA (Figure 5J) and ${ }^{99 \mathrm{~m}} \mathrm{Tc}$-sestamibi (Figure $5 \mathrm{~K}$ ) as well as time-activity curves within spinal cord ROIs (Supplemental Figure 3) showed that the higher bolus volume increased rostral distribution while also lowering local injection site exposure. Some clearance of ${ }^{111}$ In-DTPA from the IT space was detected within the kidneys and bladder, while some ${ }^{99 \mathrm{~m}} \mathrm{Tc}$-sestamibi became visible within the gastrointestinal tract over time (Figure 5, F and I, Supplemental Video 5). 
Dual imaging of ${ }^{111} \mathrm{In}-\mathrm{DTPA}$ and ${ }^{99 \mathrm{~m}} \mathrm{Tc}$-sestamibi also demonstrated the differential behavior of these agents upon intracisternal dosing into the cisterna magna. When $10 \mu \mathrm{l}$ of an ${ }^{111} \mathrm{In}$-DTPA/ ${ }^{99 \mathrm{~m}} \mathrm{Tc}$-sestamibi mixture was delivered into the cisterna magna, both agents filled the basal intracranial cisterns and most of the subarachnoid spaces surrounding the brain within the first 15 minutes (Figure 6A). With ${ }^{111}$ In-DTPA, some signal was observed across the cribriform plate as well as in the kidneys within 15 minutes. Over time, ${ }^{111}$ In-DTPA traveled caudally down the spinal column, reaching the thoracic and lumbar regions within 2 hours and 6 hours after infusion, respectively (Figure 6, A and B). ${ }^{99 \mathrm{~m} T c \text {-sestamibi again }}$ demonstrated strong local retention near the injection site and did not spread along the neuraxis over time (Figure 6, A and C).

${ }^{111} \mathrm{In}$-DTPA and ${ }^{99 \mathrm{~m}} \mathrm{Tc}$-sestamibi were both expected to undergo CSF-ISF exchange and enter the brain parenchyma along the glymphatic paravascular routes described recently (17). To investigate the respective brain parenchyma-penetrating properties of coinjected ${ }^{111} \mathrm{In}$-DTPA and ${ }^{99 \mathrm{~m}} \mathrm{Tc}$-sestamibi, magnified views of cranial SPECT-CT images from the intracisternal experiments and distribution profiles of the compounds were compared with respect to the 3D model of the cranial subarachnoid space that we developed in Figure $2 \mathrm{G}$. Beginning at 2 hours after injection, and more prominently by 6 hours, ${ }^{111}$ In-DTPA entered brain parenchyma from the CSF space (Figure 6D). ${ }^{99 \mathrm{~m}} \mathrm{Tc}$-sestamibi filled the major cranial CSF cisterns within the first 15 minutes after infusion and remained concentrated there as well as close to the injection site, without demonstrating significant movement into brain parenchyma over time (Figure 6D). Neither ${ }^{111}$ In-DTPA nor ${ }^{99 \mathrm{~m}} \mathrm{Tc}$-sestamibi showed any brain uptake when given by the i.v. route (Supplemental Figure 4). These results highlight the importance of cellular/tissue-binding affinity on penetration of drugs from the CSF into the ISF of the brain parenchyma.

\section{Discussion}

We have developed imaging approaches to study several key parameters that may underlie the high variability of IT drug therapy (5-7). CSF volume and anatomy are key parameters not typically measured in IT drug-dosing practice. Our observations suggest that, given the wide human variability of these parameters (39), measuring and normalizing for these parameters in the clinic may allow for patient-specific dose optimization. Adjusting the IT dose and bolus volume respective to an individual's MRI-estimated CSF volume may produce more predictable therapeutic responses. Areas of CSF space expansion in the spine and brain predispose adjacent CNS tissues to having greater overall exposure to IT-dosed drugs, and this should be figured into neuraxial safety and efficacy considerations. Drug delivery via the IT route may be particularly advantageous for reaching CNS tissues adjacent to these spaces.

We observed independent efflux routes from the spinal and the cranial portions of the rat IT space to the periphery and also confirmed drainage of delivered molecules across the cribriform plate and into cervical lymph nodes by live imaging. Small hydrophilic molecules exited rapidly, either predominantly $\left(\mathrm{Na}^{125} \mathrm{I},{ }^{99} \mathrm{mcO}_{4},{ }^{111} \mathrm{InCl}_{3}\right)$ or in part $\left({ }^{111} \mathrm{In}-\mathrm{DTPA},{ }^{99 \mathrm{~m}} \mathrm{Tc}-\mathrm{DMSA}\right)$, from the IT space, while ${ }^{123 / 125} \mathrm{I}-\mathrm{HSA}$ showed a very slow exchange with plasma. The exact mechanisms underlying the differential clearance of these molecules from the IT space were not addressed by this work but may include differences in passage through arachnoid granulations, into CNS parenchymal tissue and across the $\mathrm{BBB}$, or through meningeal and nasal lymphatic channels that lead to plasma via lymph nodes and lymphatic ducts (19-29). Since we did not observe signal entering the cerebral ventricles, passage of molecules across the choroid plexus was considered a less likely contributor. ${ }^{99 \mathrm{~m}} \mathrm{Tc}$-DMSA bound more tightly to CSF proteins than ${ }^{111} \mathrm{In}$-DTPA and was also slower to clear from the IT space, suggesting that adjusting drug protein binding might significantly modify retention within the IT compartment.

The strong influence of increasing the bolus volume on enhancing neuraxial exposure of both small and large molecules while reducing local injection site exposure points to this as an easily adjustable parameter for enhancement and optimization of IT dosing. The initial spread of the bolus upon injection was typically followed by a slower, steady bidirectional movement of molecules through the IT compartment at an average rate of approximately $1.5 \mathrm{~cm} / \mathrm{h}$, which likely represents the influence of mechanical convective forces produced by cardiac pulsation and respiration $(12,13,40)$.

We have developed ${ }^{99 \mathrm{~m}} \mathrm{Tc}$-sestamibi and ${ }^{186} \mathrm{Re}$-sestamibi as imaging tools for demonstrating the impact of high drug-tissue affinity on IT drug behavior. Results from studies with ${ }^{99 \mathrm{~m}} \mathrm{Tc}$-sestamibi suggest that high CNS tissue affinity will favor local IT drug retention while markedly reducing neuraxial spread. This retention can be overcome to some extent by employing larger bolus volumes. Since tissue 
accumulation of ${ }^{99 \mathrm{~m}} \mathrm{Tc}$-sestamibi is driven by mitochondrial membrane potential, the IT use of this imaging probe could also serve as a useful biomarker for assessing CNS mitochondrial function.

Using multiple clinically available molecular imaging tracers as drug surrogates in combination with dynamic dual-isotope imaging, we have quantitatively demonstrated that the regional CNS tissue exposure of injected molecules is affected by a combination of factors, including IT space anatomy, CSF dynamics, CSF clearance routes, drug molecule chemical properties, and the chosen injection site and volume of the bolus. Taken together, our data suggest that IT drug therapy can be enhanced by adjusting the protein- and tissue-binding affinity of administered drugs and by tailoring the bolus volume relative to an individual's CSF volume, as measured by MRI.

\section{Methods}

\section{Study design}

This is an experimental study performed in rats that was designed in order to develop molecular imaging-based tools to study drug delivery within the CSF-containing IT space. These tools were used to define parameters that can be manipulated in order to optimize drug exposure to specific CNS targets following lumbar drug administration with direct translation to patients. Sample sizes were determined in order to achieve statistical significance of quantified data while also balancing the desire to minimize the number of animals used as well as the high cost of conducting imaging studies. Data exclusion criteria were used in order to eliminate animals from further analyses that had obvious CSF leaks or subdural biodistribution of the injectate immediately following IT injection, as visualized by SPECT-CT. Experimental replicates are stated below.

\section{Animal care}

Approximately 10-week-old Sprague-Dawley rats (Charles River Laboratory) were kept on a 12-hour-light/ dark cycle. Rodent chow and water were available ad libitum. During all imaging and surgical procedures, animals were initially anesthetized with $4 \%-5 \%$ isoflurane $/ 0.5-11 / \mathrm{min}$ oxygen, and anesthesia was maintained with $1 \%-2 \%$ isoflurane $/ 0.51 / \mathrm{min}$ oxygen.

\section{MRI}

Imaging was conducted using a Bruker Biospec 70/30 USR (Bruker Corporation) with a 7 tesla MRI magnet. Images were acquired from $n=6$ female Sprague-Dawley rats using a 2D T2-weighted RARE sequence. The imaging parameters were as follows: $\mathrm{TR}=6,237 \mathrm{~ms}$, TE effective $=32 \mathrm{~ms}$, RARE factor $=6, \mathrm{TI}=2,083$ $\mathrm{ms}$, orientation $=$ sagittal, number of slices $=25$, slice thickness $=0.75 \mathrm{~mm}$, FOV $=5.5 \mathrm{~cm} \times 8 \mathrm{~cm}$, and resolution $=210 \mu \mathrm{m} \times 310 \mu \mathrm{m}$. Images were acquired with and without inversion pulse in 3 sections along the length of the rat spinal cord, with some overlap. Images with and without inversion were subtracted to yield a subtraction image with high signal in the CSF-containing subarachnoid spaces. For CSF within the lateral ventricles and cranial cisterns, a connected thresholding segmentation algorithm was applied to the subtracted image to segment CSF-containing spaces from the surrounding tissue. For CSF contained within the spinal column, a connected thresholding segmentation method was applied to the original MRI data to segment the spinal cord ROI from the rest of the surrounding tissue. The CSF space was defined by eroding the spinal cord ROI by one voxel in all directions. The subtracted image was used to confirm segmentation of the CSF space and to account for an overlap of the FLAIR images.

\section{Block-face cryotomography}

An IT-cannulated male Sprague-Dawley rat $(n=1)$ was placed under isoflurane anesthesia and administered a $75-\mu 1$ IT bolus of black India ink. The animal was euthanized 46 minutes after injection by inhalation overdose of $\mathrm{CO}_{2}$. In a separate experiment, $n=2$ female Sprague-Dawley rats were anesthetized with isoflurane, implanted with an IT catheter, and then injected with ${ }^{111}$ In-DTPA $(320.3 \pm 25.2 \mu \mathrm{Ci})$ in 30 $\mu 1$ followed immediately by $40 \mu \mathrm{l}(0.01 \mathrm{mg} / \mu \mathrm{l})$ of EBD. SPECT imaging was performed at 2 hours after injection to confirm successful delivery of the injectate, and at 2.5 hours after injection animals were euthanized. After euthanasia, all carcasses were frozen in a dry ice/hexane bath before being embedded in $2 \%$ carboxymethylcellulose. The tissue was sectioned using a Leica 3600 Cryomicrotome (Leica Biosystems) with the temperature maintained at approximately $-20^{\circ} \mathrm{C}$. High-resolution white-light optical images of 
the whole rat body were acquired every $25 \mu \mathrm{m}$ (555 total images). For videos, a sequence of image registration, resampling, and histogram-matching steps were used to build 3D volumes from a series of individual white-light images of sections. Preprocessed images were loaded into VivoQuant software (inviCRO LLC) to create fly-through animations and 3D models of the rat spinal column and to estimate CSF volume by application of blue color detectors.

\section{SPECT tracer labeling}

${ }^{111} \mathrm{InCl}_{3}$ was purchased from Hot Shots Nuclear Medicine. $\mathrm{Na}^{123} \mathrm{I}$ was purchased from Nordion Inc. At the time of receipt, ${ }^{111} \mathrm{InCl}_{3}$ radioactivity concentration was $0.85-0.90 \mathrm{GBq} / \mathrm{ml}$ and $\mathrm{Na}^{123} \mathrm{I}$ radioactivity concentration was $4.15-5.43 \mathrm{GBq} / \mathrm{ml}^{9}{ }^{99} \mathrm{TcO}_{4}$ was purchased from Cardinal Health at a radioactivity concentration of $2.20 \mathrm{GBq} / \mathrm{ml}$.

${ }^{123} \mathrm{I}-H S A /^{125} \mathrm{I}-H S A$. Precoated iodination tubes (Life Technologies) were wet with $1 \mathrm{ml}$ Tris iodination buffer $(25 \mathrm{mM}$ Tris- $\mathrm{HCl}, 0.4 \mathrm{NaCl}, \mathrm{pH} 7.5)$ and decanted. $100 \mu \mathrm{l}$ of the Tris iodination buffer and $20 \mu \mathrm{l}$ of $\mathrm{Na}^{123} \mathrm{I}$ ( $25 \mathrm{mCi}$, Nordion) or $\mathrm{Na}^{125} \mathrm{I}(\sim 2.7 \mathrm{mCi}$, Perkin Elmer) were added to each tube. Following a 20-minute (for ${ }^{123} \mathrm{I}$-HSA) or 9-minute (for ${ }^{125} \mathrm{I}$-HSA) activation period, $1.20-1.50 \mathrm{mg}$ of human serum albumin and the activated iodine were added to a microcentrifuge tube and mixed by gentle shaking for 45 minutes. The reaction was then terminated by the addition of 20 to $50 \mu 1$ of tyrosine scavenging buffer ( $2 \mathrm{mg} / \mathrm{ml}$ in Tris buffer) followed by mixing and incubation for 5 minutes. The reaction mixture was then purified by centrifugation through an Amicon 10K centrifugal filter (EMD Millipore) and analyzed by HPLC or iTLC for chemical purity. Radiochemical purity of the resulting products was $>94 \%$ and specific activity ranged from $3.7 \mathrm{GBq} / \mathrm{g}$ to $165.4 \mathrm{GBq} / \mathrm{g}$ at the end of synthesis.

${ }^{99 m} T c-D T P A$. Approximately 120 to $150 \mu \mathrm{g}$ of diethylenetriaminepentaacetic dianhydride (DTPAA, Sigma-Aldrich) was dissolved in $0.10 \mathrm{ml}$ of $0.9 \%$ saline purged with argon. $0.1 \mathrm{ml}$ of ${ }^{99 \mathrm{~m}} \mathrm{Tc}$ sterile saline solution (Cardinal Health) was mixed with $5 \mu \mathrm{g}$ of stannous chloride (Sigma-Aldrich) dissolved in $50 \mu 1$ of $0.9 \%$ saline solution and incubated at room temperature for 30 minutes. The resulting product was analyzed by iTLC with radiochemical purity $>95 \%$ and a specific activity from 0.22 to $0.33 \mathrm{GBq} / \mu \mathrm{mol}$ at the end of synthesis.

${ }^{111}$ In-DTPA. ${ }^{111}$ In-DTPA was purchased from Hot Shots Nuclear Medicine or radiolabeled onsite. The radioactivity concentration of ${ }^{111} \mathrm{In}-\mathrm{DTPA}$ purchased from Hot Shots was $2.50-4.00 \mathrm{GBq} / \mathrm{ml}$. Radiolabeling was performed by indium chelation using two different approaches. In the first approach, ${ }^{111} \mathrm{InCl}_{3}$ was neutralized using $0.6 \mathrm{M}$ ammonium acetate buffer. Following neutralization, approximately $2 \mathrm{mCi}$ of ${ }^{111} \mathrm{InCl}_{3}$ was transferred into a cryovial containing DTPA for a total volume of $37 \mu 1$. $100 \mu 1$ of $0.6 \mathrm{M}$ ammonium acetate buffer was added to $5 \mathrm{mg}$ of DTPA $(5 \mathrm{mg} / \mathrm{ml})$ in the bottom of dry Nunc tubes. $24 \mu \mathrm{l}(1,200 \mu \mathrm{g})$ of the stock DTPA $(50 \mathrm{mg} / \mathrm{ml})$ was transferred to a $1.8-\mathrm{ml}$ cryotube. $2 \mathrm{mCi}$ of ${ }^{111}$ In-DTPA was transferred into the $1.8-\mathrm{ml}$ cryotube containing DTPA. The product was incubated at $37^{\circ} \mathrm{C}$ for 0.5 hours and mixed gently at 30 minute intervals. Each sample of final purified material was then injected on the HPLC (SEC isocratic Phenomenex column). The purified ${ }^{111} \mathrm{In}-\mathrm{DTPA}$ samples were transferred into the HPLC analytic sample vials, and appropriate volumes were aliquoted onto the analytical column for HPLC analysis. Purity as determined by HPLC was $100 \%$. Specific activity was $61.68 \mathrm{GBq} / \mathrm{g}$ and radioactivity concentration was $0.62 \mathrm{GBq} / \mathrm{ml}$.

In the second approach, ${ }^{111} \mathrm{InCl}_{3}$ sterile solution from Mallinckrodt Pharmaceuticals was purchased through Cardinal Health. DTPAA (CAS 23911-26-1) was purchased from Sigma-Aldrich. Sodium acetate trace select grade (a product of Fluka Analytical) was purchased from Sigma-Aldrich. Approximately 200 to $250 \mu \mathrm{g}$ of DTPAA was taken up within approximately 50 to $100 \mu 1$ of a $0.5 \mathrm{M}$ sodium acetate buffer ( $\mathrm{pH}$ 5.5). To the resulting solution was added to 50 to $100 \mu 1$ of ${ }^{111} \mathrm{InCl}_{3}$ solution and was heated to $40^{\circ} \mathrm{C}$ for 1 hour. The resulting product was analyzed by iTLC and used as is. Radiochemical purity ranged from $95 \%$ to $99 \%$, and specific activity ranged from 0.13 to $0.16 \mathrm{GBq} / \mu \mathrm{mol}$ at the end of synthesis. Radioactivity concentration was $0.37-0.46 \mathrm{GBq} / \mathrm{ml}$.

${ }^{99 m} T c-D M S A$. RENOCIS ${ }^{99 m}$ Tc-DMSA kits were purchased from IBA CIS Bio International. ${ }^{99 \mathrm{~m} T c-}$ DMSA was prepared according to kit instructions: ${ }^{99} \mathrm{TcO}_{4}(40 \mathrm{mCi}$ in $1 \mathrm{ml})$ was added to the kit, the glass vial was shaken, and the reaction was allowed to incubate for 15 minutes at room temperature. Radiochemical purity was $>99 \%$ as determined by iTLC. Radioactivity concentration of the final product used for imaging was $1.48 \mathrm{GBq} / \mathrm{ml}$.

${ }^{99 m}$ Tc-sestamibi. ${ }^{99 \mathrm{~m}} \mathrm{Tc}$-sestamibi was purchased from Hot Shots Nuclear Medicine. Radioactivity concen- 
tration on the day of imaging was $0.57-4.75 \mathrm{GBq} / \mathrm{ml}$.

${ }^{186}$ Re-sestamibi. $\left[{ }^{186} \mathrm{Re}\right]$ as $\left[\mathrm{ReO}_{4}\right]^{-1}$ was received from the Missouri University Research Reactor as alumina perrhenate in water $(19.6 \mathrm{GBq} / \mathrm{ml}$ and $87.6 \mathrm{GBq} / \mathrm{mg})$. The radioisotope was reacted with freshly prepared stannous chloride and neat 1-isocyano-2-methoxy-2-methylpropane (MIBI) in refluxing ethanol. The crude reaction was purified by solid-phase exclusion via a Sep-Pak C-18 cartridge and formulated in $10 \%$ ethanol/saline. Radiochemical purity was tested by HPLC ( $>90 \%)$ with ${ }^{99 m}$ Tc-MIBI as an identity reference. Radioactivity concentration of the final product delivered for imaging was $111 \mathrm{MBq} / \mathrm{ml}$, and specific activity was $37 \mathrm{GBq} / \mathrm{mmol}$.

\section{IT lumbar and cisternal injections}

Lumbar needle stick injections. Once fully anesthetized, the lower dorsal region of the animal was shaved, and the L5-L6 region was scrubbed with a chlorhexidine wipe (2\%). A small incision was made between the L5 and L6 vertebrae. The needle of a 27-gauge 0.5 - $\mathrm{ml}$ tuberculin syringe was inserted between the L5 and L6 vertebrae, and correct positioning of the needle in the IT space was determined by a tail-flick response of the animal. Upon confirmation of correct positioning, the bolus (consisting of either dosing solution alone or dosing solution separated from a saline flush by an air bubble within the syringe) was slowly injected.

Cannulated lumbar injections. Following anesthesia, a 1.0- to $2.0-\mathrm{cm}$ incision was made over the anterior pelvic and lumbar region followed by a $1.0-\mathrm{cm}$ incision in the muscle capsule immediately lateral to the dorsal spinal process of the 6th lumbar vertebra. The bones of the 6th lumbar vertebra were visualized by blunt dissection. A guide cannula needle assembly was positioned near the anterior aspect of the 6th lumbar vertebra and pushed into the intravertebral space. A catheter with the stylet wire was inserted into the guide cannula, and the guide cannula was then removed leaving the catheter in place. The catheter was angled down at an acute angle to the spinal canal and the end was forced approximately $0.3 \mathrm{~cm}$ into the spinal canal. The stylet wire was removed approximately $2.5 \mathrm{~cm}$ from the tip of the catheter, and the catheter was advanced $2 \mathrm{~cm}$ into the spinal canal. The guide wire was then completely withdrawn, and the catheter was sutured in place and flushed with a small amount of sterile saline. Correct placement of the catheter was confirmed by backflow of CSF into catheter when the guide wire was withdrawn or when the needle was removed. Animals were then placed in the prone position and injected slowly with the desired bolus volume of injectate.

Intracisternal injections. Once fully anesthetized, the animals were placed in ventral recumbency on a collection platform that was developed to optimize the angle of the neck for access to the cisterna magna $\left(\sim 70^{\circ}\right)$. The cervical region of the back was shaved and scrubbed with a chlorhexidine wipe $(2 \%)$. The needle of a 27 -gauge $0.5-\mathrm{ml}$ Tuberculin syringe was inserted posterior to the base of the skull along the cervical midline. Correct positioning was determined by backflow of a small amount of CSF into the syringe, followed by slow injection of the dosing solution.

\section{SPECT-CT imaging}

${ }^{123} \mathrm{I}$-HSA $(\sim 750 \mu \mathrm{Ci})$ was injected IT in a $10-\mu \mathrm{l}$ bolus $(n=4)$ and in a $50-\mu \mathrm{l}$ bolus $(n=5)$. Na ${ }^{123} \mathrm{I}(408 \mu \mathrm{Ci})$ was injected IT in a $10-\mu \mathrm{l}$ bolus $(n=1)$ and i.v. $(1,505 \mu \mathrm{Ci})$ in a $100-\mu \mathrm{l}$ bolus $(n=1) .{ }^{111} \mathrm{InCl}_{3}(310 \mu \mathrm{Ci})$ was injected IT in a $10-\mu \mathrm{l}$ bolus $(n=1)$ and i.v. $(1,538 \mu \mathrm{Ci})$ in a $100-\mu \mathrm{l}$ bolus $(n=1) .{ }^{99 \mathrm{~m}} \mathrm{TcO}_{4}(86 \mu \mathrm{Ci})$ was injected IT in a $30-\mu 1$ bolus followed by a $40-\mu 1$ saline flush $(n=1)$ and i.v. $(859 \mu \mathrm{Ci})$ in a $200-\mu 1$ bolus $(n=1)$. ${ }^{99}$ Tc-sestamibi $(\sim 630 \mu \mathrm{Ci})$ was injected IT in a $10-\mu \mathrm{l}$ bolus $(n=2)$ and i.v. $(1,381 \mu \mathrm{Ci})$ in a $100-\mu \mathrm{l}$ bolus $(n=1) .{ }^{111} \mathrm{In}$-DTPA $(\sim 800 \mu \mathrm{Ci})$ and ${ }^{99 \mathrm{~m}} \mathrm{Tc}$-sestamibi $(\sim 800 \mu \mathrm{Ci})$ were mixed together and coinjected $\mathrm{IC}$ in a $10-\mu \mathrm{l}$ bolus $(n=4)$ and IT in a $30-\mu \mathrm{l}$ bolus followed by a 40- $\mu \mathrm{l}$ saline flush $(n=5) .{ }^{111} \mathrm{In}-\mathrm{DTPA}(\sim 800 \mu \mathrm{Ci})$ and ${ }^{99 \mathrm{~m} T c-s e s t a m i b i}$ $(300-800 \mu \mathrm{Ci})$ were also mixed together and coinjected IT in a $20-\mu 1$ bolus $(n=6) .{ }^{111}$ In-DTPA $(\sim 200 \mu \mathrm{Ci})$ and ${ }^{99 \mathrm{~m}} \mathrm{Tc}$-DMSA $(\sim 400 \mu \mathrm{Ci})$ were mixed together and coinjected IT in a $30-\mu 1$ total volume followed by a $40-\mu 1$ saline flush $(n=1) .{ }^{186} \mathrm{Re}$-sestamibi $(8 \mu \mathrm{Ci})$ was injected IT in a $40-\mu \mathrm{l}$ bolus $(n=2)$.

SPECT-CT scanning was completed after tracer administration on the NanoSPECT/CT imaging system or nanoScan SPECT/CT imaging system (Mediso USA). Image data were acquired at 0 to 1 (four 9-minute scans), 2, and 6 hours (one 30-minute scan) after injection. High-resolution SPECT scans were performed using rat 9-pinhole apertures (1.4- to 2.5-mm diameter). Energy windows selected for each isotope were $155.6-186.4$ and $220.5-269.5 \mathrm{keV}$ for ${ }^{111} \mathrm{In} ; 127.9-153.2 \mathrm{keV}$ for ${ }^{99 \mathrm{~m}} \mathrm{Tc}$; $143.9-174.9$ keV for ${ }^{123} \mathrm{I}$; $24.3-29.7 \mathrm{keV}$ for ${ }^{125} \mathrm{I}$; and $54.9-67.1$ and $123.48-150.92$ for ${ }^{186}$ Re. Following each SPECT acquisition, a CT was acquired to serve as an anatomical reference during imaging analysis. SPECT scans were reconstructed using HiSPECT software (Scivis $\mathrm{GmBH}$ ). 
ROIs were defined using a combination of manual and automated segmentation based on both SPECT and CT. The CSF ROI refers to both the spinal cord and CSF space regions (i.e., the subarachnoid space). In addition, advanced analysis techniques were used to divide the CSF ROI into cervical, thoracic, and lumbar subregions by identifying the split points for each region based on anatomy. The radioactivity concentration (radioactivity/volume) in each ROI was normalized to the injected radioactivity dose, and it was assumed that $1 \mathrm{~cm}^{3}=1 \mathrm{~g}$ of tissue. Image analysis was performed using the VivoQuant software (inviCRO LLC).

\section{Cranial CSF model}

SPECT data sets resulting from imaging of intrathecally injected animals were evaluated qualitatively for signal in the cranial CSF. Selected data sets were registered to a common space (along with a single CT data set) using a series of rigid and affine registrations (ITK). Registered data sets were averaged, following an intensity normalization step, to produce a mean cranial CSF uptake image. Brain tissue and cranial CSF ROIs were generated on the mean cranial CSF uptake image using a combination of automated and manual methods. The resulting ROI mask underwent morphological operations to spatially smooth and impose left-right symmetry.

\section{Planar scintigraphy}

A mixed solution of either ${ }^{99} \mathrm{TcO}_{4}(\sim 750 \mu \mathrm{Ci})$ and ${ }^{125} \mathrm{I}-\mathrm{HSA}(\sim 300 \mu \mathrm{Ci})$ or ${ }^{99 \mathrm{~m}} \mathrm{Tc}-\mathrm{DMSA}(\sim 400 \mu \mathrm{Ci})$ and ${ }^{111}$ In-DTPA $(\sim 200 \mu \mathrm{Ci})$ was injected via cannulated lumbar puncture as described above (bolus volume $=$ $25 \mu \mathrm{l}$ plus $15-\mu 1$ saline flush separated from the dose mixture by an air bubble). Animals were then immediately placed in the prone position on the bed of the nanoScan SPECT/CT imaging system (Mediso USA). A dynamic planar scintigraphy scan of 2 hours total length was acquired. The acquisition consisted of three hundred 1-second frames, ninety 10 -second frames, and one hundred 60 -second frames, followed by a single 600 -second frame from 120 to 130 minutes. Energy windows of 126.5 to $154.6 \mathrm{keV}$ and 24.3 to 29.7 $\mathrm{keV}$ were used for ${ }^{99 \mathrm{~m}} \mathrm{Tc}$ and ${ }^{125} \mathrm{I}$. For planar imaging with ${ }^{111} \mathrm{In}$, images were acquired using energy windows centered around emission peaks at 173 and $245 \mathrm{keV}$. Images for the higher energy 220.86- to 269.94$\mathrm{keV}$ window showed an unacceptably high level of background due to septal penetration of the low-energy collimator. Therefore, only images in the lower-energy 158.5 - to $184.1-\mathrm{keV}$ window were analyzed in this study. The energy windows chosen for analysis of the dual radioisotopes showed some overlap of ${ }^{99 \mathrm{~m}} \mathrm{TC}$ into the ${ }^{111}$ In energy window (see Supplemental Figure 2). To correct for this overlap in the planar scintigraphy data (Figure 3, B, F, and G, and Supplemental Video 3), the ${ }^{111}$ In-DTPA images were smoothed with a 4.0-mm full-width-and-half-maximum Gaussian approach, multiplied by 0.29 (29\% spillover, as measured from existing spectra), and then subtracted from the ${ }^{99 \mathrm{~m}} \mathrm{Tc}-\mathrm{DMSA}$ images.

To reduce imaging noise, counts in all frames shorter than 60 seconds were summed to create 120 total frames of 60-second duration. ROIs of the spine, brain, kidneys, bladder, and stomach were delineated on an image of all summed counts over 120 minutes using a combination of manual and automated segmentation. In each ROI, the average count rate in counts per minute was measured within each 60 -second frame to derive the local kinetics of each tracer.

\section{Tracer protein binding}

In triplicate, each radiotracer $(40 \mu \mathrm{l})$ was mixed with substrate $(40 \mu \mathrm{l})$ in an eppendorf tube and incubated at $37^{\circ} \mathrm{C}$ for 60 minutes. Briefly, substrates (rat CSF, human CSF, and HSA) were sourced as follows: rat CSF was collected from animals via stereotactic insertion of a 29-gauge needle into the cisterna magna and collection into a syringe under anesthesia, human CSF was purchased (Lee Biosolutions), and a 5\% solution of HSA was made by dilution of HSA (Sigma-Aldrich) into distilled water. Protein binding was assessed using size exclusion chromatography with Sephadex MicroSpin G-50 columns (GE Healthcare). Columns were prepared by centrifuging at 2,000 $\mathrm{g}$ for 1 minute. A $25-\mu 1$ aliquot of each incubated substrate/sample mixture was then added, and the columns were centrifuged again at 2,000 $\mathrm{g}$ for 2 minutes. The radioactivity from both the collected eluate and the column-bound fraction were measured with a dose calibrator, and this ratio was used to calculate the percentage of protein-bound radiotracer.

\section{Whole-body autoradiography}

At 2.5 hours after injection of $495 \mu \mathrm{Ci}^{111} \mathrm{In}-\mathrm{DTPA}(n=1)$ or $360 \mu \mathrm{Ci}{ }^{99 \mathrm{~m}} \mathrm{Tc}$-sestamibi $(n=1)$ IT in a $30-\mu \mathrm{l}$ bolus followed by a $40-\mu 1$ saline flush, animals were euthanized and carcasses were frozen in hexane/dry 
ice. The head was segmented from the rest of the body and embedded in a $2 \%$ carboxymethylcellulose matrix and placed in a freezer set to maintain $-70^{\circ} \mathrm{C}$ to $-90^{\circ} \mathrm{C}$ until the tissue was frozen solid. Transverse sections were cut using a Leica CM3600 cryomacrotome (Leica Biosystems) at $20-\mu \mathrm{m}$ thickness. Sections were mounted, exposed to phosphor image screens, and scanned using the Storm 860 image acquisition system (Molecular Dynamics Inc., now GE Healthcare).

\section{Microautoradiography}

At 6 hours after injection of $8 \mu \mathrm{Ci}^{111}$ Re-sestamibi $(n=2)$ IT in a $40-\mu 1$ bolus followed by a $30-\mu 1$ saline flush, animals were euthanized, and brains and spinal cords were resected. Tissues were frozen and cryoembedded in OCT. Under safelight conditions in a dark room, superfrost plus gold slides (Thermo Scientific) were precoated by dipping slides in nuclear track material (NTB) emulsion (Carestream), diluted 1:1 with distilled water, and allowed to air-dry overnight. 10- $\mu \mathrm{m}$-thick OCT-embedded tissue sections were cut and thaw-mounted onto the precoated slides. The slides were air-dried for 5 minutes, rinsed with PBS buffer, incubated for 5 minutes in freshly made D19 Developer (Eastman Kodak) diluted 1:3 with distilled water, rinsed, incubated for 5 minutes with Kodafix Fixative solution diluted 1:3 in distilled water, and rinsed again. The slides were then briefly counterstained for 15 seconds in methylene blue-basic fuchsin, before being mounted with a coverslip. The slides were imaged using a Leica DMRE microscope (Leica Camera AG) using either bright-field settings or a dark-field condenser for detection of silver grains.

\section{Statistics}

For IT injections, animals that received a missed injection, as defined by the presence of significant levels of radioactive tracer in the subdural, rather than IT, space, were excluded from analyses. For protein-binding experiments, statistical significance between groups was determined using 1-way ANOVA followed by Tukey's multiple comparisons test. All time-activity curves were plotted as mean \pm SD. Data from individual time points were analyzed by unpaired 2-tailed Student's $t$ tests, and 2-way repeated-measures ANOVA analyses were performed between groups. All statistical analyses were performed using Prism 6 software (GraphPad Software). A $P$ value less than 0.05 was considered significant.

\section{Study approval}

Experiments reported in this paper were performed at two sites: MPI Research (Mattawan, Michigan, USA) and inviCRO LLC. All animal experiments were conducted in full compliance with MPI Research or inviCRO LLC Institutional Animal Care and Use Committee (IACUC) policies and procedures, which follow the recommendations of the Guide for the Care and Use of Laboratory Animals (8th ed. National Academy Press, 2011). Prior to the commencement of experiments, all experimental procedures involving animals were reviewed and approved by the IACUC of the site performing the experiment.

\section{Author contributions}

DAW planned experiments, analyzed data, generated the figures, and wrote the manuscript. JYH, JMS, KDO, MDS, ML, and TW planned the experiments, carried out the experiments, analyzed the data, and edited the manuscript. JH planned the experiments, analyzed the data, and edited the manuscript. AV planned the experiments, analyzed the data, and wrote the manuscript.

\section{Acknowledgments}

We would like to thank numerous members of the staff at inviCRO LLC for their contributions, including project management, project execution, and data analysis efforts that have not adequately been captured in the remaining text, as the data presented in this manuscript are the result of an extensive and lengthy group effort. We would also like to thank the staff at the Translational Imaging Center at MPI Research, especially Dustin and Joshua Kentala for expert technical assistance. Additionally, we would like to thank Curt Mazur at ISIS Pharmaceuticals for providing training for the lumbar catheterization injection protocol. Biogen Inc. provided funding for all experiments presented in this manuscript. The authors had full editorial control of the manuscript and provided final approval of all content.

Address correspondence to: Ajay Verma, Experimental Medicine, 14 Cambridge Center, Cambridge, Massachusetts 02142, USA. Phone: 617.679.4311; E-mail: Ajay.Verma@biogen.com. 
1. Chen Y, Liu L. Modern methods for delivery of drugs across the blood-brain barrier. Adv Drug Deliv Rev. 2012;64(7):640-665.

2. Zhang ET, Inman CB, Weller RO. Interrelationships of the pia mater and the perivascular (Virchow-Robin) spaces in the human cerebrum. J Anat. 1990;170:111-123.

3. Iliff JJ, et al. A paravascular pathway facilitates CSF flow through the brain parenchyma and the clearance of interstitial solutes, including amyloid beta. Sci Transl Med. 2012;4(147):147ra11.

4. Krahn V. The pia mater at the site of the entry of blood vessels into the central nervous system. Anat Embryol (Berl). 1982;164(2):257-263

5. Sallerin-Caute B, Lazorthes Y, Monsarrat B, Cros J, Bastide R. CSF baclofen levels after intrathecal administration in severe spasticity. Eur J Clin Pharmacol. 1991;40(4):363-365.

6. Shafer SL, Eisenach JC, Hood DD, Tong C. Cerebrospinal fluid pharmacokinetics and pharmacodynamics of intrathecal neostigmine methylsulfate in humans. Anesthesiology. 1998;89(5):1074-1088.

7. Hocking G, Wildsmith JA. Intrathecal drug spread. Br J Anaesth. 2004;93(4):568-578.

8. Miller TM, et al. An antisense oligonucleotide against SOD1 delivered intrathecally for patients with SOD1 familial amyotrophic lateral sclerosis: a phase 1, randomised, first-in-man study. Lancet Neurol. 2013;12(5):435-442.

9. Penn RD, Kroin JS, York MM, Cedarbaum JM. Intrathecal ciliary neurotrophic factor delivery for treatment of amyotrophic lateral sclerosis (phase I trial). Neurosurgery. 1997;40(1):94-99.

10. Rigo F, et al. Pharmacology of a central nervous system delivered 2'-O-methoxyethyl-modified survival of motor neuron splicing oligonucleotide in mice nonhuman primates. J Pharmacol Exp Ther. 2014;350(1):46-55.

11. Papisov MI, Belov VV, Gannon KS. Physiology of the intrathecal bolus: the leptomeningeal route for macromolecule and particle delivery to CNS. Mol Pharm. 2013;10(5):1522-1532.

12. Hsu Y, Hettiarachchi HD, Zhu DC, Linninger AA. The frequency and magnitude of cerebrospinal fluid pulsations influence intrathecal drug distribution: key factors for interpatient variability. Anesth Analg. 2012;115(2):386-394.

13. Hettiarachchi HD, Hsu Y, Harris TJ Jr, Penn R, Linninger AA. The effect of pulsatile flow on intrathecal drug delivery in the spinal canal. Ann Biomed Eng. 2011;39(10):2592-2602.

14. Hladky SB, Barrand MA. Mechanisms of fluid movement into, through and out of the brain: evaluation of the evidence. Fluids Barriers CNS. 2014;11(1):26.

15. Brinker T, Stopa E, Morrison J, Klinge P. A new look at cerebrospinal fluid circulation. Fluids Barriers CNS. $2014 ; 11: 10$.

16. Iliff JJ, et al. Brain-wide pathway for waste clearance captured by contrast-enhanced MRI. J Clin Invest. 2013;123(3):1299-1309.

17. Yang L, et al. Evaluating glymphatic pathway function utilizing clinically relevant intrathecal infusion of CSF tracer. $J$ Transl Med. 2013;11:107.

18. Calias $\mathrm{P}$, et al. CNS penetration of intrathecal-lumbar idursulfase in the monkey, dog and mouse: implications for neurological outcomes of lysosomal storage disorder. PLoS One. 2012;7(1):e30341.

19. d'Avella D, Cicciarello R, Albiero F, Andrioli G. Scanning electron microscope study of human arachnoid villi. J Neurosurg. 1983;59(4):620-626.

20. Kida S, Yamashima T, Kubota T, Ito H, Yamamoto S. A light and electron microscopic and immunohistochemical study of human arachnoid villi. J Neurosurg. 1988;69(3):429-435.

21. Weed LH. Studies on Cerebro-Spinal Fluid. No. II: the theories of drainage of cerebro-spinal fluid with an analysis of the methods of investigation. J Med Res. 1914;31(1):21-49.

22. Zakharov A, Papaiconomou C, Djenic J, Midha R, Johnston M. Lymphatic cerebrospinal fluid absorption pathways in neonata sheep revealed by subarachnoid injection of Microfil. Neuropathol Appl Neurobiol. 2003;29(6):563-573.

23. Zakharov A, Papaiconomou C, Johnston M. Lymphatic vessels gain access to cerebrospinal fluid through unique association with olfactory nerves. Lymphat Res Biol. 2004;2(3):139-146.

24. Johnston M, Zakharov A, Koh L, Armstrong D. Subarachnoid injection of Microfil reveals connections between cerebrospinal fluid and nasal lymphatics in the non-human primate. Neuropathol Appl Neurobiol. 2005;31(6):632-640.

25. Johnston M, Zakharov A, Papaiconomou C, Salmasi G, Armstrong D. Evidence of connections between cerebrospinal fluid and nasal lymphatic vessels in humans, non-human primates and other mammalian species. Cerebrospinal Fluid Res. 2004;1(1):2.

26. Koh L, Zakharov A, Nagra G, Armstrong D, Friendship R, Johnston M. Development of cerebrospinal fluid absorption sites in the pig and rat: connections between the subarachnoid space and lymphatic vessels in the olfactory turbinates. Anat Embryol (Berl). 2006;211(4):335-344.

27. Aspelund A, et al. A dural lymphatic vascular system that drains brain interstitial fluid and macromolecules. J Exp Med. 2015;212(7):991-999.

28. Tarasoff-Conway JM, et al. Clearance systems in the brain-implications for Alzheimer disease. Nat Rev Neurol. 2015;11(8):457470 .

29. Louveau A, et al. Structural functional features of central nervous system lymphatic vessels. Nature. 2015;523(7560):337-341.

30. Koh L, Zakharov A, Johnston M. Integration of the subarachnoid space and lymphatics: is it time to embrace a new concept of cerebrospinal fluid absorption? Cerebrospinal Fluid Res. 2005;2:6.

31. Nagra G, Koh L, Zakharov A, Armstrong D, Johnston M. Quantification of cerebrospinal fluid transport across the cribriform plate into lymphatics in rats. Am J Physiol Regul Integr Comp Physiol. 2006;291(5):R1383-R1389.

32. Chiu C, et al. Temporal course of cerebrospinal fluid dynamics and amyloid accumulation in the aging rat brain from three to thirty months. Fluids Barriers CNS. 2012;9(1):3.

33. Roy D, Steyer GJ, Gargesha M, Stone ME, Wilson DL. 3D cryo-imaging: a very high-resolution view of the whole mouse. Anat Rec (Hoboken). 2009;292(3):342-351.

34. Kida S, Pantazis A, Weller RO. CSF drains directly from the subarachnoid space into nasal lymphatics in the rat. Anatomy, histology and immunological significance. Neuropathol Appl Neurobiol. 1993;19(6):480-488.

35. Ichimura T, Fraser PA, Cserr HF. Distribution of extracellular tracers in perivascular spaces of the rat brain. Brain Res. 1991;545(1-2):103-113.

36. Huhmer AF, Biringer RG, Amato H, Fonteh AN, Harrington MG. Protein analysis in human cerebrospinal fluid: Physiological aspects, current progress and future challenges. Dis Markers. 2006;22(1-2):3-26. 
37. Lee BF, et al. Evaluation of Tc-99m (V) DMSA binding to human plasma proteins. Kaohsiung J Med Sci. 2008;24(1):1-9.

38. Rehling M, Nielsen LE, Marqversen J. Protein binding of 99Tcm-DTPA compared with other GFR tracers. Nucl Med Commun. 2001;22(6):617-623.

39. Grant R, et al. Human cranial CSF volumes measured by MRI: sex and age influences. Magn Reson Imaging. 1987;5(6):465-468.

40. Chen L, Beckett A, Verma A, Feinberg DA. Dynamics of respiratory and cardiac CSF motion revealed with real-time simultaneous multi-slice EPI velocity phase contrast imaging. Neuroimage. 2015;122(1):281-287. 